\title{
Region Mathematics-a New Direction in Mathematics: Part-1
}

\section{Ranjit Biswas}

Department of Computer Science \& Engineering, Faculty of Engineering \& Technology, Jamia Hamdard University, Hamdard Nagar, New Delhi, India

\section{Email address:}

ranjitbiswas@yahoo.com

To cite this article:

Ranjit Biswas. Region Mathematics-a New Direction in Mathematics: Part-1. Pure and Applied Mathematics Journal. Vol. 5, No. 3, 2016, pp. 39-59. doi: 10.11648/j.pamj.20160503.11

Received: March 23, 2016; Accepted: April 13, 2016; Published: April 25, 2016

\begin{abstract}
In this paper the author introduces a new direction in Mathematics called by "Region Mathematics" to the world mathematicians, academicians, scientists and engineers. The purpose of developing 'Region Mathematics' is not just for doing a generalization of the existing rich volume of classical Mathematics, but it has automatically happened so by this work. To introduce the 'Region Mathematics', we begin here with introducing three of its initial giant family members: Region Algebra, Region Calculus and Multi-dimensional Region Calculus. Three more of its initial giant family members: Theory of Objects, Theory of A-numbers (Number Theory) and Region Geometry will follow in the sequel work. The development of the subject 'Region Mathematics' is initiated from its zero level for all its initial giant family members. The subject is expected to grow very fast with time to take its own shape, and it will surely cater to all branches of Science, Engineering, and others wherever an element of mathematics needs to be done. With the introduction of Region Mathematics, all existing branches of mathematics will get wide horizontal shifts in the academic universe of science, mathematics, engineering, social science, statistics, etc. with many more alternative new approaches and new thoughts.
\end{abstract}

Keywords: Region, Object, Calculus Space, Region Calculus, Complete Region

\section{Introduction}

In this paper the author introduces a new direction in 'Mathematics' for the world mathematicians, academicians, scientists and engineers. By 'Mathematics' we mean a vast family consisting of a large number of giant members viz. Algebra, Number Theory, Arithmetic, Geometry, Trigonometry, Calculus, Mechanics, Astronomy,... etc. to list a few only out of many. In fact this work is not just about a new direction, but about a generalization of all the classical directions of existing huge volume of 'Mathematics' in an integrated universal way. That is the reason why we call it a universal mathematics. It will be insufficient if we just say that this is a new direction. It is basically a new vast direction consisting of many new directions. This is the first work on "Region Mathematics" and quite obviously it starts here from zero level. The 'Region Mathematics' is a newly discovered mathematics which has been justified to be viewed as a universal mathematics of super giant volume.
The existing huge volume of mathematics is just a part of it; although apparently it seems that the existing volume of mathematics has been almost sufficiently supporting the demands of the world mathematician, world scientists, world statisticians, and world engineers in their all type of mathematical works. The work may apparently seem to be too simple at the first readings, because it is fact that it is simple and of very fundamental nature. Because of its very simple initial nature, the readers may have to take patience to read the materials till end, even if some of the theories/propositions happen to be unacceptable or debatable initially. The direction is launched by discovering the algebraic structure "Region" first of all, on submitting sufficient justification behind the genuine and mandatory need to discover it; then introducing the "Region Calculus". In the sequel of this work done in Part-2 [7], we introduce "Theory of Objects", then introduce a new language of the "Theory of Numbers", and also a new topic "Region Geometry". But with the introduction of few of its giant family members, it is the beginning of the super-giant 
"Region Mathematics", and at this moment it is at baby stage. The purpose of developing the super-giant 'Region Mathematics' is not just for doing a kind of generalization of the existing rich volume of classical Mathematics, but it has automatically happened so in its initial growth here. The complete content of this paper can be well studied without referring to our previous work in [3-6], because the present work is a major revised and updated version of all these previous work.

The idea knocked my mind while I unearthed a peculiar fact that most of the simple and useful results, equalities, identities, formulas etc. of elementary algebra, which are commonly practiced at secondary school level of mathematics and ofcourse at all levels of higher mathematics, are not valid (can not be verified) in groups, rings, modules, fields, linear spaces, algebra over a field, associative algebra over a field, and even not in 'division algebra', i.e. are not valid (can not be verified) in any existing standard algebraic system alone, in general, by virtue of their respective definitions and properties. I became very much curious to scan the issue thoroughly. Initially I got confused, I got puzzled, I could not find out 'what is the reason, what is wrong or where is the deficit in the existing volume of theories?'. I completely doubted only upon myself as my main area of research is not 'Algebra'. I then revisited my college life favorite books of Herstein, Jacobson, Lang, Waerden, etc. from my bookshelves. In fact I tried a permutation/combination of the various existing algebraic structures to make out a possible identity of a platform algebraic system in which all the daily-useful results, identities, formulas, laws, etc. of elementary algebra can be verified. Finally I became fully confident that there is an excellent something lying hidden so far in the subject 'Algebra'. I unearthed the hidden beauty, which is a highly ornamented and beautiful algebraic system which I call by 'Region'. A special attention needs to be given to explore and study this hidden algebraic system considering its unique potential to provide a complete and sound platform, a minimal platform, on which the simple and useful results, equalities, identities, formulas etc. of elementary algebra can be verified (recognized to be valid). This attention was missing in the subject Algebra so far because of the reason that the serious importance and strength of this hidden algebraic system was so far not identified, although a huge and rich volume of literature is available on various algebraic systems. In quest of identifying this leading platform, the new algebraic system called by "Region" is developed independently with a unique identity in Section-3. Considering the enormous unique potential of "Region Algebra" to give license to the mathematicians to practice the existing simple and useful results, equalities, identities, formulas etc. of elementary algebra, world can not ignore the deserving and genuine claim of "Region Algebra" to have a self independent identity for it. It is because of the reason that the world has given due identity to the other algebraic systems including Division Algebra each of which has a much lower potential compared to "Region Algebra" in the core and application areas of mathematics from school to higher level. Many of the properties and results of the set $\mathrm{R}$ of real numbers are being so far used by the world mathematicians, assuming $\mathrm{R}$ to be a division algebra, but without knowing that they are actually using the 'region' properties of $\mathrm{R}$. This is justified in this paper in details that the properties of division algebra is not sufficient for them to solve many (or most) of the mathematical problems. Fortunately the division algebra $\mathrm{R}$ is also a region algebra, and thus world mathematicians did not face any problem so far while discovering and developing various topics of mathematics (viz. Theory of Numbers, Geometry, Calculus, etc.) by exploiting fluently the infinite number of interesting properties of $\mathrm{R}$ but without looking at the actual identity of the algebraic platform upon which the results stand valid. In the progress of mathematics in many branches, the set $\mathrm{R}$ of real numbers probably is being always pre-assumed to be a division algebra, and this paper justifies that this preassumption is not sufficient i.e. does not give license to use many simple results, formula, rules, identities etc. freely and fluently. It is a Region Algebra at minimum. Region Mathematics will reform the classical mathematics in an optimal generalized shape. It will be a serious mistake if we can not realize the unique superiority of 'Region Mathematics' as it is the absolute generalization of the classical mathematics and of a subject of unlimited applications. Without 'Region Mathematics' the existing huge volume of mathematics will surely lack its deserving materials to get its final shape of universal nature. Certainly Region Mathematics will provide the world scientists of all areas a further scope and thinking ability to analyze their works in a more significant and generalized way via a new gate of huge broadband, in pursuance of better results, in pursuance of unearthing an extended coverage of the problems under their study. The subject "Region Mathematics" is at its baby stage today, and will grow very fast at its own natural pace if the initial literature presented here be understood by the world. There is a possibility that readers may acquire reasons to ignore "Region Mathematics" because of its birth happened with simple type of mathematics and while they will come across very simple and elementary terms like: $(a x+b y)^{2}$, Associativity, Crossmultiplication, Componendo \& Dividendo, etc. in this article during the course of its analysis. Because on the other side of the mind it is fact that our scientists are roaming around Mars, or even around Pluto in this solar system and ofcourse roaming around the universe (or multiverse, if exists), with the existing super-giant volume of mathematics!. We proceed then to introduce a new direction in Calculus called by "Region Calculus" by defining "Calculus Space" in Section4. It is observed that the existing calculus of Newton and Leibnitz is a particular case of region calculus. It is claimed that there could be requirements of new generalized types of calculus to see the universe more precisely, and if it is so then there must be a path consisting of genuine steps which will ensure whether in a certain environment a new calculus can be developed or not. If it can be developed, then the 
question arises on 'how to develop'. It is also explained here that the philosophy of developing a new calculus is same as that of the originators Newton and Leibnitz. Then we extend the concept of calculus space by introducing multidimensional calculus space and the corresponding multidimensional region calculus. The properties of region algebra are very important as this is the 'minimal algebra' which justifies free and fluent practice of elementary as well as higher algebra. This important caliber of regions having the unique property to be qualified as the 'minimal algebra' in the sense of giving a kind of driving license to the world mathematicians, the caliber which is not possessed by groups, rings, modules, fields, linear spaces, algebra over a field, associative algebra over a field, and even not by 'division algebra' or by any existing standard algebraic system alone, in general, by virtue of their respective definitions and properties. This important identification, probably the most important issue in the subject 'Algebra' and one of the most important issues in Mathematics, was missing so far in any past literature of algebra or mathematics, and thus it is surely a unique algebra of absolute integrated nature and super power. With the introduction of Region Mathematics, all existing branches of mathematics can be provided their siblings with the progress in future research works, in order to explore the academic universe of science, mathematics, engineering, social science, statistics, etc. with many more alternative new approaches and new thoughts.

\section{Recollecting the Standard Definitions of 'F-algebra', 'Associative Algebra over a Field F' and 'Division Algebra'}

Throughout the discussion in this paper, the following standard definitions are followed for 'F-Algebra', 'Associative Algebra over a field F', and for 'Division Algebra' [1, 2, 10-23, 26, 28]:-

\section{1. 'Algebra over a Field F' (F-ALGEBRA)}

An 'Algebra over a field F' (or, a F-Algebra) is a vector space A over $\mathrm{F}$ equipped with a compatible notion of multiplication

$$
(\mathrm{a} \bullet \mathrm{x}) *(\mathrm{~b} \bullet \mathrm{y})=(\mathrm{a} . \mathrm{b}) \bullet(\mathrm{x} * \mathrm{y}), \forall \mathrm{a}, \mathrm{b} \in \mathrm{F} \text { and } \forall \mathrm{x}, \mathrm{y} \in \mathrm{A} \text {. }
$$

\section{2. 'Associative Algebra over a Field F'}

An 'Associative Algebra over a field $F$ ' is a vector space over $\mathrm{F}$ which also allows the multiplication of vectors in a distributive and associative manner, having bilinearity of the multiplication.

\section{3. 'Division Algebra'}

A 'Division Algebra' $\left(\mathrm{D},+,{ }^{*}\right)$ is a set $\mathrm{D}$ together with two binary operators + and $*$ such that it is a unit ring and $(\mathrm{D}$ $\left.\left\{0_{D}\right\},{ }^{*}\right)$ forms a group. A division algebra D allows division operation by non-zero elements, but $\mathrm{D}$ need not be commutative with respect to its multiplication operation. Even the compatible notion of multiplication may or may not be satisfied in D. A division Algebra is also called by "division ring" or "skew field". For example, the set $\mathrm{R}$ of real numbers, the set $\mathrm{C}$ of complex numbers, the Cayley algebra, the set of quaternions, etc. are few examples of division algebra.

\section{Region Algebra}

Algebra is regarded as one of the most beautiful branches of mathematics and it is about finding the unknowns. It tastes to be both dry and juicy. More precisely Algebra is about converting the real life problems of engineering, science, mathematics, social science, etc. into equations/inequations and then solving them to understand the unknowns. Algebra is taught from primary/secondary school level as one of the subjects in the course-curriculum popularly known as "Elementary Algebra" which develops the thinking capabilities, specifically logic, patterns, problem solving, deductive and inductive reasoning of the students. It is shown by a number of examples here that most of the simple and useful results, identities/equalities, formulas or algebraic expressions or equations (commonly practiced at secondary school level of mathematics) of elementary algebra are not valid (i.e. can not be computed/verified) in general in any of the existing standard algebraic systems alone: viz. in a group alone, or in a ring alone, or in a field alone, or in module, linear space, algebra over a field, in an associative algebra over a field, or in a division algebra alone, etc. By the phrase: "the result is valid in the algebraic system A", we mean here that the result can be successfully computed and established/verified in the algebraic system A. Consequently, it is unearthed that there was a major gap lying hidden so far in the existing literature on the subject "Algebra". To fill-up this gap a new algebraic system called by "Region" is introduced independently in a unique way. The huge potential and strength of this powerful algebraic system is lying in the fact that it validates the simple results, equalities, identities, formulas etc. of elementary algebra which are commonly practiced at secondary school level of mathematics, whereas most of them can not be computed/verified in general in any of the existing standard algebraic systems alone. The issue happened to my mind by chance only, by luck, with no prior plan or thinking of mine to proceed for developing a new algebraic system. Region alone provides the minimal platform on which all elementary algebraic computations practiced by students, teachers, mathematicians, scientists, engineers, etc. are done. Such a complete and sound platform for practicing 'elementary algebra' can not be provided by any existing algebraic system alone like: group, ring, module, field, linear space, algebra over a field, associative algebra over a field, division algebra, etc. in general. This important fact was hidden so far to the algebraists, and has been now unearthed here. With this philosophy, it can be realized that all the existing classical algebraic systems are weaker than the algebraic system 'region' in terms of application potential and 
caliber. An initial development of the theory of regions is done with a lot of characterizations, establishing a number of important properties of the regions.

\subsection{Justification Behind the Genuine Need to Define/Identify a New Algebraic System and to Introduce It Uniquely in an Independent Way}

A system consisting of a non-null set $\mathrm{S}$ and one or more nary operators on the set $\mathrm{S}$ is called an algebraic system, denoted by the notation $\left(\mathrm{S}, \mathrm{O}_{1}, \mathrm{O}_{2}, \ldots, \mathrm{O}_{\mathrm{r}}\right)$ where $\mathrm{O}_{\mathrm{i}}, \mathrm{i}=$ $1,2, \ldots, \mathrm{r}$, are operators on $\mathrm{S}$. An algebraist can define an infinite number of new algebraic systems. The objective of the work in this section is not just for the sake of defining a new algebraic system, but to recognize and identify a major gap of the subject 'Algebra' lying hidden so far in the existing vast literature developed till date.

In this section we show that the existing algebraic systems viz. groups, rings, modules, fields, linear spaces, algebra over a field, associative algebra over a field, division algebra or any existing standard algebraic system, by virtue of their definitions, are not sufficient to provide a sound and complete environment/platform or algebraic right to the mathematicians for performing many simple algebraic computations, for establishing many useful and simple identities or equalities of two algebraic expressions, and for establishing many useful algebraic results/solutions etc. of elementary algebra; although many of these results/equalities/identities are very much well known even to the secondary school students, and being practiced fluently by the students, teachers, academicians, engineers, scientists, etc in the world. Fortunately none is arriving at any contradiction too (even if the scientists do excellent work like sending satellite to Mars or Pluto), the reasons for which has been unearthed and clarified with various sub-reasons stated at every section till the end of the content of this paper and of the work [7].

Let us begin here with a collection of few cases or issues (out of infinite number) on the various standard algebraic systems: groups, rings, modules, fields, etc. These cases (five cases) are mentioned below for the sake of instance only, although they are no doubt very simple and obvious cases to any algebraist. But special attention of the readers is required on the situations presented in Case-4. Then we justify the genuine needs for identifying a new kind of atomic, well complete, sound and unique algebraic system in an independent way with its self-identity.

Few Cases (by examples):

Case-1: If an expression like $\mathrm{x} \oplus \mathrm{y} / \mathrm{z}$ is known to be a valid expression in an algebraic system A (while let us suppose that nothing is known to us at this stage about the identity or about the properties of the algebraic system A), where $\mathrm{x}, \mathrm{y}, \mathrm{z} \in \mathrm{A}$, then one can say that $\mathrm{A}$ is not just a group or a ring in general; however it could be a Division Algebra or any algebra which is also a division algebra.

Case-2: If an expression like $\mathrm{x} \oplus 2 . \mathrm{y} \oplus 5 . \mathrm{z}$ is a valid expression in an algebraic system A while nothing is known to us at this stage about the identity or about the properties of the algebraic system $\mathrm{A}$, where $\mathrm{x}, \mathrm{y}, \mathrm{z} \in \mathrm{A}$ (assuming that associativity property hold $\operatorname{good}$ in A over the operator $\oplus$ ), then one can say that A is not just a group or ring or a field, in general. However, it could be a linear space over the field $\mathrm{R}$ of real numbers, or something else which is also a linear space over the field $\mathrm{R}$ of real numbers.

Case-3: If an expression like $\mathrm{x}+2 . \mathrm{y} \odot \mathrm{z}$ is a valid expression in an algebraic system $A$ where $x, y, z \in A$, then one can immediately say that A can not be just a group or a ring or a field or a linear space in general. However, it could be an 'Associative Algebra over a field', or something else.

Consider the equality (identity): $(x+y)^{2}=x^{2}+2 .(x * y)+$ $\mathrm{y}^{2}$ which is an absurd equality (as it can not be verified) in general in a group or in a ring/module or in a field or in a linear space or in an associative algebra over a field. However, it can be well verified in some 'algebra over some field'. Here it may be noted that the LHS of this equality can be evaluated in a ring or in a field, but not the RHS (assuming that the notation $\mathrm{t}^{2}$ stands for the expression $\mathrm{t} * \mathrm{t}$ as usual).

Now consider three interesting situations in Case-4 below: Case-4 (three examples here):

Example 3.1

A very simple example from elementary algebra, very frequently used by the secondary school students, is the equality (identity) I of type given by

$$
\left(\frac{2}{3}\right) \cdot\left(\frac{x}{y}\right)=\left(\frac{2 \cdot x}{3 \bullet y}\right)
$$

but it is not valid i.e. can 'not be verified' in general in a group, ring, module, field, linear space, 'algebra over a field (i.e. F-algebra)', 'associative algebra over a field', Division Algebra, or in any existing standard brand of algebraic system alone.

Justification

It is because of the reason that:

(1) since division operations are involved, it can not be a simple 'F-algebra' alone.

(2) on the other hand, if it is not a'F-algebra' but a simple division algebra $\mathrm{D}$ alone, then the following are fact:-

(i) the LHS expression $\left(\frac{2}{3}\right) \bullet\left(\frac{x}{y}\right)$ can be well written to be equal to the expression $\left(2 \cdot \frac{1}{3}\right) \bullet\left(\mathrm{x} * \mathrm{y}^{-1}\right)$ in the division algebra $\mathrm{D}$,

(ii) but the expression $\left(2 \cdot \frac{1}{3}\right) \bullet\left(\mathrm{x}^{*} \mathrm{y}^{-1}\right)$ can not be written in the division algebra $\mathrm{D}$, by virtue of its definition and own properties, to be equal to the expression $(2 \bullet \mathrm{x}) *\left(\frac{1}{3} \bullet y^{-1}\right),($ see Section 2$)$.

(iii) although, it is true that in the division algebra $\mathrm{D}$, by virtue of its definition and own properties, 


$$
(2 \bullet \mathrm{x}) *\left(\frac{1}{3} \bullet y^{-1}\right) \text { is equal to the expression }\left(\frac{2 \bullet x}{3 \bullet y}\right) .
$$

Consequently, in a simple division algebra $\mathrm{D}$, the expression $\left(\frac{2}{3}\right) \bullet\left(\frac{x}{y}\right)$ can not be equal to $\left(\frac{2 \bullet x}{3 \bullet y}\right)$, in general, except in some particular cases where few additional conditions or properties need to be also satisfied by the division algebra $\mathrm{D}$ as its additional qualifications (although a 'division algebra' by definition does not need to satisfy these additional requirements of properties). Fortunately, in many of these cases the objects $\mathrm{x}, \mathrm{y}$ are real numbers and by default the set $\mathrm{R}$ of real numbers satisfies some additional interesting properties beyond the properties possessed by division algebra alone or any existing brand of algebraic system alone. Consequently, although there is no single brand of existing algebraic system alone which has the capability to provide the set $\mathrm{R}$ these additional requirements of properties, the mathematicians have not been facing any problem and have not been getting any incorrect results or contradictory results because of some interesting reasons which have been unearthed and analyzed in this section in details. This is a very useful, very powerful, very important (probably most important fact in Algebra) and going to be very prominent fact which is missing in the existing literatures on Algebra.

It can also be observed that in this simple elementary expression, three multiplication operators '.', '• ', and '*' are involved.

Example 3.2

With the same argument as in Example 3.1 above, it can be observed that if an equality (in fact an identity) I of type given by

$$
\left((a \bullet x) \oplus \frac{1}{b \bullet y}\right)^{2}=a^{2} \cdot x^{2} \oplus \frac{1}{b^{2} \bullet y^{2}} \oplus\left(\frac{2 a}{b}\right) \cdot\left(\frac{x}{y}\right)
$$

is known to be a valid identity (i.e. can be computed and verified) in an algebraic system $A$ where $x, y \in A, a$ and $b$ are members (scalars) of some field F, then it can be observed that all of the following statements are not true in general (unless few additional properties are satisfied beyond their respective definitions):

a. A is just a group alone, or a ring, module, field, linear space

b. A is just an 'algebra over a field F' (F-algebra)

c. A is just an 'associative algebra over a field'

d. A is just a 'Division Algebra' alone

e. A is any standard existing brand of algebraic system, in general.

Example 3.3

By a careful observation it can also be seen that even a simple computation of 'cross-multiplication' $\mathrm{C}$ of secondary school level elementary algebra like:

if $\frac{2 \bullet x}{7 \bullet y}=\frac{5 \bullet z}{3 \bullet t}$ then $6^{\bullet} \mathrm{x} * \mathrm{t}=35^{\bullet} \mathrm{y} * \mathrm{z}$ (and conversely)

is not valid in a division algebra, by virtue of its definition and its own properties, or in any standard algebraic system.

\section{Brief Justification:}

The justification is in fact similar to what made in Example 3.1

(1) since division operations are involved, it can not be a simple 'F-algebra' alone.

(2) on the other hand, if it is not a 'F-algebra' but a simple division algebra $\mathbf{D}$ alone, then the following are fact:-

$$
\begin{aligned}
& \text { Suppose that } \frac{2 \bullet x}{7 \bullet y}=\frac{5 \bullet z}{3 \bullet t} \\
& \text { Or, }(2 \bullet x)(7 \bullet y)^{-1}=(5 \bullet z)(3 \bullet t)^{-1}
\end{aligned}
$$

which can not yield the result $6 \bullet \mathrm{x} * \mathrm{t}=35 \bullet \mathrm{y} * \mathrm{z}$ in a division algebra, by virtue of its definition and its own properties.

Similarly, for another example, it may be carefully seen that a very simple square identity I like: $\left(\frac{3 \bullet x}{7 \bullet y}\right)^{2}=\frac{9 \bullet x^{2}}{49 \bullet y^{2}}$ can 'not be verified' (i.e. not valid) in general in a group, ring, module, field, linear space, 'algebra over a field (i.e. Falgebra)', 'associative algebra over a field', Division Algebra, or any standard algebraic system (assuming that division by the zero element is not allowed).

Then, the immediate question that arises to an algebraist is: "What algebraic system is A?" Or "What could be the minimal algebraic system in which the above identities I or the above cross multiplication result $\mathrm{C}$ are valid?". Or "What algebraic system the identities $\mathrm{I}$ or these results can be verified in?". For a possible answer, an algebraist has to think of a permutation/combination of the various existing algebraic structures to make out a possible identity of A. But, he might seek to make a unique identity for this algebraic system A to define it in an independent and atomic way, and then to study the various properties of A, various results valid on A, highlighting its unique importance/role in Algebra compared to all other standard algebraic systems. It is because of the reason that this algebraic system $\mathrm{A}$ is supposed to be the most appropriate and needful minimal platform for practicing the problems from elementary algebra of secondary school level to higher algebra, compared to any other existing standard algebraic system, in general. Thus the role of this algebraic system to the world mathematicians is much more than any other existing algebraic system. Consequently there a genuine need to identify that algebraic system, which is hidden so far, unrecognized so far, but a very powerful algebraic system in the sense that it can provide the actual and minimal base-platform for practicing the subjects elementary algebra and higher algebra, compared to what the existing algebraic systems can provide. It is fact that algebraic identities/equalities, formulas or algebraic expressions or equations involving two kinds of multiplication operators and one (or two) kind of addition operator are very common and frequent in the study of mathematics, science subjects, engineering subjects from secondary school level to higher levels(!). Such kind of computations are probably the most frequent and of routine type exercises at secondary school level to college/university and research level of study. For example, in matrix theory, 
expressions with two kinds of multiplication operators (matrix multiplication and scalar multiplication) and with one kind of addition operator (matrix addition) like $\mathrm{A}+2 \mathrm{BCD}^{-1}-$ $5 \mathrm{XY}^{-2}$ where $\mathrm{A}, \mathrm{B}, \mathrm{C}, \mathrm{D}, \mathrm{X}, \mathrm{Y}$ are real square matrices of order $\mathrm{n}$, are very common and frequent.

\subsection{Introducing a New Algebraic System 'Region'}

It has been observed that there is a genuine need to introduce a new algebraic system having unique self-identity in order to provide a minimal but sufficient platform based upon which the elementary algebra or higher algebra can be fluently practiced by the mathematicians with valid algebraic right and driving license.

\subsubsection{Region}

Consider a non-null set A equipped with three binary operators $\oplus, *$ and $\bullet$ such that for a given field $(\mathrm{F},+,$.$) , the$ following three conditions are satisfied:-

(i) $(\mathrm{A}, \oplus, *)$ forms a field,

(ii) $(\mathrm{A}, \oplus, \bullet)$ forms a linear space over the field $(\mathrm{F},+,$.$) ,$ and

(iii) A satisfies the property of "Compatibility with the scalars of the field $F "$ A.

i.e. $(\mathrm{a} \bullet \mathrm{x}) *(\mathrm{~b} \bullet \mathrm{y})=(\mathrm{a} . \mathrm{b}) \bullet(\mathrm{x} * \mathrm{y}) \forall \mathrm{a}, \mathrm{b} \in \mathrm{F}$ and $\forall \mathrm{x}, \mathrm{y} \in$

Then the algebraic system $\left(\mathrm{A}, \oplus,{ }^{*}, \bullet\right)$ is called a Region over the field $(\mathrm{F},+,$.$) .$

If there is no confusion, we may simply use the notation $A$ to represent the region $(\mathrm{A}, \oplus, *, \bullet)$, for brevity.

We now study the various interesting properties of a region A.

Two Fields: Inner Field and Outer Field (Base Field)

The field $(\mathrm{A}, \oplus, *)$ is called the "inner field" of the region $\left(\mathrm{A}, \oplus,{ }^{*}, \bullet\right)$; and the field $(\mathrm{F},+,$.$) of the linear space (\mathrm{A}, \oplus$, - ) is called the "outer field" or the "base field" of the region $(\mathrm{A}, \oplus, *, \bullet)$.

\subsubsection{Three Multiplication Operators}

First Multiplication Operator, Second Multiplication Operator, and Third Multiplication Operator (or Base Multiplication Operator)

The sequence of the three operators “ $\oplus$ ", “*”, and “•" appearing in the notation $(\mathrm{A}, \oplus, *, \bullet)$ representing the region $\mathrm{A}$ is important in the sense that the operator "**" of the region A which is the multiplication operator of the inner field (A, $\left.\oplus,{ }^{*}\right)$ is called the "first multiplication" operator of the region A. The operator "•" of the region A which is the multiplication operator of the linear space $(\mathrm{A}, \oplus, \bullet)$ is called the "second multiplication" operator of the region $\mathrm{A}$. The multiplication operator ". " of the base field F is called the "third multiplication" operator or the "base multiplication" operator of the region A.

\subsubsection{Two Addition Operators: First Addition Operator and Third Addition Operator}

The operator " $\oplus$ " of the region $(\mathrm{A}, \oplus, *, \bullet)$ which is the addition operator of the inner field $\left(\mathrm{A}, \oplus,{ }^{*}\right)$ as well as of the linear space $(\mathrm{A}, \oplus, \bullet)$ is called the "first addition" operator of the region A. The operator "+" which is the addition operator of the base field $(\mathrm{F},+,$.$) is called the "third addition"$ operator or the "base addition" operator of the region A.

There is no terminology like "second addition" operator of a region $\mathrm{A}$.

Thus in a region $\mathrm{A}$, we deal with two addition operators and three multiplication operators, in general. It is obvious from the definition that a region A must have at least two elements. It may also be noted that every region is an 'algebraic system over a field', but the converse is not true in general.

As a simple instance, it could be now seen that an equality (identity) I of type given by $\left(\frac{2}{3}\right) \bullet\left(\frac{x}{y}\right)=\left(\frac{2 \bullet x}{3 \bullet y}\right)$,

which can not be verified, in general, in a group or in a ring or in a field or in a linear space or in an associative algebra over a field, or in a division algebra or even not in a simple 'algebra over a field', now can be well verified or established in the algebraic system 'region' alone, and hence obviously in any algebraic system which is at least a region.

As another simple instance, it could be now seen that an identity like

$$
\left((a \bullet x) \oplus \frac{1}{b \bullet y}\right)^{2}=a^{2} \cdot x^{2} \oplus \frac{1}{b^{2} \bullet y^{2}} \oplus\left(\frac{2 a}{b}\right) \cdot\left(\frac{x}{y}\right)
$$

which can not be verified, in general, in a group or in a ring or in a field or in a linear space or in an associative algebra over a field, or in a division algebra or even not in a simple 'algebra over a field', now can be well verified or established in the algebraic system 'region' and hence obviously in any algebraic system which is at least a region.

And it can be also be seen now that even a simple computation of 'cross-multiplication' $\mathrm{C}$ of secondary school level elementary algebra like:

if $\frac{2 \bullet x}{7 \bullet y}=\frac{5 \bullet z}{3 \bullet t}$ then $6^{\bullet} \mathrm{x} * \mathrm{t}=35^{\bullet} \mathrm{y} * \mathrm{z}$ (and conversely).

or, a very simple square identity I like: $\left(\frac{3 \bullet x}{7 \bullet y}\right)^{2}=\frac{9 \bullet x^{2}}{49 \bullet y^{2}}$

which can not be verified, in general, in a group or in a ring or in a field or in a linear space or in an associative algebra over a field, or in a division algebra or even not in a simple 'algebra over a field', now can be well verified or established in the algebraic system 'region' and hence obviously in any algebraic system which is at least a region.

A region $(\mathrm{A}, \oplus, *, \bullet)$ is defined here as an atomic algebraic system with two multiplication operators, one is internal operative and the other is with the scalars of F. This characteristic makes the algebraic system region to become the most appropriate platform for practicing and validating the results of 'elementary algebra' (and also college algebra, higher algebra etc.), compared to the standard algebraic systems like groups, rings, modules, fields, linear spaces, algebra over a field, associative algebra over a field, division algebra, etc. 
In the region $(\mathrm{A}, \oplus, *, \bullet)$ over the field $(\mathrm{F},+,$.$) , one of its$ component algebraic systems is $(\mathrm{A}, \oplus, *)$ which is a field. Thus we see that the region $\mathrm{A}$ is a commutative and also a division algebra. Besides that the other component algebraic system $(\mathrm{A}, \oplus, \bullet)$ of the region $\mathrm{A}$ is a linear space over the field F. Considering the distributive properties of the field (A, $\oplus, *)$ along with the condition(iii) mentioned in the definition of region above, it is observed that the region $\mathrm{A}$ is also a F-algebra. Thus, integrating these three facts, we can see that a region is a "commutative division F-algebra", but defined independently and uniquely with a self-identity here. An algebra satisfying only the property of 'commutative' is not sufficient to define the algebra we have been in quest here, satisfying only the properties of 'division algebra' is not sufficient to define the algebra we have been in quest here, satisfying only the properties of 'F-algebra' is not sufficient to define the algebra we have been in quest here. At minimum it must be a region. It could be any other algebra which is also a region. But the minimum platform is the region, not less. Clearly a region is not a division algebra only, but a lot of things more. However, a region can also be viewed as permutation/combination of the existing classical algebraic systems in other ways too, But considering its major role and huge importance to all the mathematicians, our purpose is to give due regards to it providing it an independent identity and clearly studying its various properties here.

Example 3.4

The region RR: the most useful region in Science, Engineering \& Other areas.

Let $\mathrm{R}$ be the set of real numbers, '+' be the ordinary addition operator in $\mathrm{R}$ and '.' be the ordinary multiplication operator in $\mathrm{R}$. Consider the field $(\mathrm{R},+,$.$) of real numbers, and$ the linear space $(\mathrm{R},+,$.$) over the field (\mathrm{R},+,$.$) . Then the$ algebraic system $(\mathrm{R},+, . .$.$) forms a region over the outer field$ $(\mathrm{R},+,$.$) .$

This region $(\mathrm{R},+, . .$.$) plays a very important role in our$ daily life computations, in particular in school level elementary algebra/arithmetic. The content of the syllabus and corresponding instructions at school level algebra is based on the platform of this region $(\mathrm{R},+, .,$.$) , not on the$ platform of any standard algebraic structure like groups, rings, fields, linear spaces, algebra over a field, associative algebra over a field, division algebra or any existing algebraic system, in general. Let us name this region $(\mathrm{R},+, . .$, in short by "RR". The region RR is the most useful region in the existing Mathematics, Science, Engineering \& Other areas.

One of the most beautiful qualifications, rich merits and strengths of the Regions is that all the following three important associative properties collectively may not be true in a simple division algebra alone, or in any standard algebraic system alone, but they are well valid in any region; although they are frequently and freely being used by the mathematicians, scientists and engineers in their any mathematical work or computation of everyday life.

\subsubsection{Three Associativity Properties of Regions}

The following three associative properties hold good in a region $\left(\mathrm{A}, \oplus,{ }^{*}, \bullet\right)$ over the field $(\mathrm{F},+,$.$) . They are called$ "No-Scalar Associative Property", "One-Scalar Associative Property" and "Two-Scalars Associative Property" respectively.

(i) $\mathrm{x} *(\mathrm{y} * \mathrm{z})=(\mathrm{x} * \mathrm{y}) * \mathrm{z}$ : (No-Scalar Associative Property)

(ii) $\mathrm{a} \bullet(\mathrm{x} * \mathrm{y})=(\mathrm{a} \bullet \mathrm{x}) * \mathrm{y}:($ One-Scalar Associative Property)

(iii) $(\mathrm{a} . \mathrm{b}) \bullet \mathrm{x}=\mathrm{a} \bullet(\mathrm{b} \bullet \mathrm{x})$ : (Two-Scalars Associative Property)

where $\mathrm{a}, \mathrm{b} \in \mathrm{F}$ and $\mathrm{x}, \mathrm{y}, \mathrm{z} \in \mathrm{A}$.

Proof:

(i) This follows by inheritance from the properties of the inner field $\left(\mathrm{A}, \oplus,{ }^{*}\right)$.

(ii) Consider the property of "Compatibility with the scalars of field $\mathrm{F}^{\prime}$ ' in the region $\left(\mathrm{A}, \oplus,{ }^{*}, \bullet\right)$ given by:

$(\mathrm{a} \bullet \mathrm{x})^{*}(\mathrm{~b} \bullet \mathrm{y})=(\mathrm{a} \cdot \mathrm{b}) \bullet\left(\mathrm{x}^{*} \mathrm{y}\right)$. Substituting $1_{\mathrm{F}}$ for $\mathrm{b}$ in the above, we get the result of 'One-Scalar Associative Property', where $1_{\mathrm{F}}$ is the unit element of the outer field ( $\mathrm{F}$, $+,$.$) .$

(iii) It follows by inheritance from the properties of the linear space $(\mathrm{A}, \oplus, \bullet)$.

NOTE 3.1 Throughout this section, the following conventions are to be assumed in the context of region (A, $\oplus, *, \bullet)$ over the field $(\mathrm{F},+,$.$) , without any confusion:- by the$ expression $\mathrm{a} \bullet \mathrm{x} \oplus \mathrm{y}$, we shall mean $(\mathrm{a} \bullet \mathrm{x}) \oplus \mathrm{y}$, not $\mathrm{a} \bullet(\mathrm{x} \oplus$ $\mathrm{y})$; the expression $\mathrm{a} \bullet \mathrm{x} * \mathrm{~b} \bullet \mathrm{y}$ means the expression $(\mathrm{a} \bullet \mathrm{x}) *$ $(\mathrm{b} \bullet \mathrm{y})$; the expression $\mathrm{a} \bullet \mathrm{x} \oplus \mathrm{b} \bullet \mathrm{y}$ means $(\mathrm{a} \bullet \mathrm{x}) \oplus(\mathrm{b} \bullet \mathrm{y})$; the expression $\mathrm{a} . \mathrm{b} \bullet \mathrm{x}$ means the expression $(\mathrm{a} \cdot \mathrm{b}) \bullet \mathrm{x}$.

\subsection{5. “Additive Identity" Element of a Region}

The additive identity element of the inner field $(\mathrm{A}, \oplus, *)$ of a region $(\mathrm{A}, \oplus, *, \bullet)$ is called the 'additive identity' element of the region $\mathrm{A}$, and is denoted by the notation $0_{\mathrm{A}}$. Obviously, the 'additive identity' element of a region $\mathrm{A}$ is unique, by virtue of inheritance from the properties of the inner field (A, $\left.\oplus,{ }^{*}\right)$. The additive identity of a region $\mathrm{A}$ is also called the 'zero element' of the region A. It is obvious that the zeroelement of the linear space $(\mathrm{A}, \oplus, \bullet)$ and the element $0_{\mathrm{A}}$, the zero element of the region $\mathrm{A}$, both are the same elements.

\subsection{6. "Multiplicative Identity" Element of a Region}

The multiplicative identity element of the inner field (A, $\left.\oplus,{ }^{*}\right)$ of a region $\left(\mathrm{A}, \oplus,{ }^{*}, \bullet\right)$ is called the 'multiplicative identity' element of the region $\mathrm{A}$ and is denoted by the notation $1_{\mathrm{A}}$. Obviously, 'multiplicative identity' element of a region $\mathrm{A}$ is unique, by virtue of inheritance from the properties of the inner field $(\mathrm{A}, \oplus, *)$. The multiplicative identity of a region $\mathrm{A}$ is also called the 'unit element' of the region $\mathrm{A}$.

\subsection{7. "Additive Inverse" of an Element of a Region}

For an element $\mathrm{x}$ of a region $(\mathrm{A}, \oplus, *, \bullet)$ over the field $(\mathrm{F}$, $+,$.$) , the 'additive inverse' of \mathrm{x}$ is defined to be that element of the region $\mathrm{A}$ which is the additive inverse of $\mathrm{x}$ in the inner field $(\mathrm{A}, \oplus, *)$, and is denoted by the notation $\sim \mathrm{x}$. Obviously, 'additive inverse' of an element of a region is 
unique, by virtue of inheritance from the properties of the inner field $(\mathrm{A}, \oplus, *)$.

\subsection{8. “Multiplicative Inverse" of an Element of a Region}

For a non-zero element $\mathrm{x}$ of a region $(\mathrm{A}, \oplus, *, \bullet)$ over the field $(\mathrm{F},+,$.$) , the 'multiplicative inverse' of \mathrm{x}$ is defined to be that element of the region $A$ which is the multiplicative inverse of $\mathrm{x}$ in the inner field $(\mathrm{A}, \oplus, *)$, and is denoted by the notation $\mathrm{x}^{-1}$. Obviously, 'multiplicative inverse' of an element of a region is unique, by virtue of inheritance from the properties of the inner field $(\mathrm{A}, \oplus, *)$. It may be observed that "multiplicative inverse" $\mathrm{x}^{-1}$ of an element $\mathrm{x}$ of a region $\mathrm{A}$ is with respect to the first multiplication operator of the region A. There is no multiplicative inverse of an element $\mathrm{x}$ of the region $\mathrm{A}$ with respect to the second multiplication operator ' $\bullet$ ' and with respect to the third multiplication operator ' '.

The following proposition on a region $(\mathrm{A}, \oplus, *, \bullet)$ over the field $(\mathrm{F},+,$.$) is obvious, being inherited from its inner field$ $\left(\mathrm{A}, \oplus,{ }^{*}\right)$ for the results (i) and (ii), and being inherited from the linear space $(\mathrm{A}, \oplus, \bullet)$ for the results (iii) and (iv).

Proposition 3.1

In a region $(\mathrm{A}, \oplus, *, \bullet)$ over the field $(\mathrm{F},+,$.$) , for \mathrm{a}, \mathrm{b} \in$ $\mathrm{F}$ and for $\mathrm{x}, \mathrm{y} \in \operatorname{region} \mathrm{A}$,

(i) if $\mathrm{x}=\mathrm{y}$, then $\mathrm{x} \oplus \mathrm{z}=\mathrm{y} \oplus \mathrm{z} \forall \mathrm{z} \in$ region $\mathrm{A}$.

(ii) if $\mathrm{x}=\mathrm{y}$, then $\mathrm{x} * \mathrm{z}=\mathrm{y} * \mathrm{z} \forall \mathrm{z} \in$ region $\mathrm{A}$.

(iii) if $\mathrm{x}=\mathrm{y}$, then $\mathrm{a} \bullet \mathrm{x}=\mathrm{a} \bullet \mathrm{y} \forall \mathrm{a} \in \mathrm{F}$.

(iv) if $\mathrm{a}=\mathrm{b}$, then $\mathrm{a} \bullet \mathrm{z}=\mathrm{b} \bullet \mathrm{z} \forall \mathrm{z} \in \operatorname{region} \mathrm{A}$.

The following result is true in region algebra.

Proposition 3.2

In a region $\left(\mathrm{A}, \oplus,{ }^{*}, \bullet\right)$ over the field $(\mathrm{F},+,$.$) , for \mathrm{a} \in \mathrm{F}$ and for $\mathrm{x}\left(\neq 0_{\mathrm{A}}\right), \mathrm{y} \in$ region $\mathrm{A}$, if $\mathrm{y} * \mathrm{x}=\mathrm{a} \bullet \mathrm{x}$ then $\mathrm{y}=\mathrm{a} \bullet 1_{\mathrm{A}}$.

Proof: We have $\mathrm{y} * \mathrm{x}=\mathrm{a} \bullet \mathrm{x}$

$$
\therefore(\mathrm{y} * \mathrm{x}) * \mathrm{x}^{-1}=(\mathrm{a} \bullet \mathrm{x}) * \mathrm{x}^{-1}
$$

Applying 'No-scalar Associative Property' on LHS and 'One-scalar Associative property' on RHS, we get

$$
\begin{aligned}
& \mathrm{y} *\left(\mathrm{x} * \mathrm{x}^{-1}\right)=\mathrm{a} \bullet\left(\mathrm{x}^{*} \mathrm{x}^{-1}\right) \\
& \therefore \mathrm{y}=\mathrm{a} \bullet 1_{\mathrm{A}} \text {. Hence proved. }
\end{aligned}
$$

\subsubsection{Four Types of 'Division' in a Region Algebra}

Let $(\mathrm{A}, \oplus, *, \bullet)$ be a region over the field $(\mathrm{F},+,$.$) . There$ are four types of division can be performed in this algebraic system which are mentioned below. For all these four types of division, we use a common notation/style like $\frac{\text { numerator }}{\text { deno min ator }}$, (assuming that there is no confusion).

Division Type-(i) Division of an 'element of the region A' by another 'element of the region $\mathrm{A}$ '

$\forall \mathrm{x}, \mathrm{y}\left(\neq 0_{\mathrm{A}}\right) \in \operatorname{region} \mathrm{A}$, the division of the element $\mathrm{x}$ by the non-zero element $\mathrm{y}$ is denoted by the notation $\frac{x}{y}$, and is defined by $\frac{x}{y}=\mathrm{x} * \mathrm{y}^{-1}$ or $\mathrm{y}^{-1} * \mathrm{x}$ (as they are commutative). Replacing $\mathrm{x}$ by $1_{\mathrm{A}}$ and $\mathrm{y}$ by $\mathrm{x}$ in the above, we get the result $\frac{1_{A}}{x}=\mathrm{x}^{-1}\left(\right.$ where $\left.\mathrm{x} \neq 0_{A}\right)$.
Division Type-(ii) Division of 'an element of the region A' by 'an element of the outer field $\mathrm{F}$ '

$\forall \mathrm{x} \in \mathrm{A}$ and $\forall \mathrm{a}(\neq 0 \mathrm{~F}) \in \mathrm{F}$, the division of the region element $\mathrm{x}$ by the field element $\mathrm{a}$ is denoted by $\frac{x}{a}$, and is defined by $\frac{x}{a}=\mathrm{a}-1 \bullet \mathrm{x}$. (It may be noted that an expression like $\mathrm{x} \bullet \mathrm{a}-1$ is not valid here in general, except for some particular regions). Replacing a by $1 \mathrm{~F}$, we get the result $\frac{x}{1_{F}}$ $=\mathrm{x}$.

Division Type-(iii) Division of 'an element of the outer field $\mathrm{F}$ ' by 'an element of the region A'

$\forall \mathrm{a} \in \mathrm{F}$ and $\forall \mathrm{x}(\neq 0 \mathrm{~A}) \in \mathrm{A}$, the division of the field element a by the region element $\mathrm{x}$ is denoted by $\frac{a}{x}$, and is defined by $\frac{a}{x}=\mathrm{a} \bullet \mathrm{x}^{-1}$. (It may be noted that an expression like $\mathrm{x}^{-1} \bullet \mathrm{a}$ is not valid here). Replacing a by $1 \mathrm{~F}$, we get the result $\frac{1_{F}}{x}=\mathrm{x}^{-1}$.

NOTE 3.2

From the two equalities $\mathrm{x}^{-1}=\frac{1_{A}}{x}$ and $\frac{1_{F}}{x}=\mathrm{x}^{-1}($ where $\mathrm{x} \neq$ $0_{\mathrm{A}}$ ), we get the result $\frac{1_{A}}{x}=\frac{1_{F}}{x}$. But using Proposition 3.2 we observe that a cancellation-law is not applicable to this result here, and consequently the equality $1_{\mathrm{A}}=1_{\mathrm{F}}$ does not emerge to be true (in general, except for some particular regions).

Division Type-(iv) Division of 'an element of the outer field $F$ ' by another 'element of the outer field $F$ '.

In the field $(\mathrm{F},+,$.$) , it is known (in field theory) that \forall \mathrm{a}, \mathrm{b}$ $\left(\neq 0_{\mathrm{F}}\right) \in \mathrm{F}$, the division of the element a by the non-zero element $\mathrm{b}$ is denoted by the notation $\frac{a}{b}$, and is defined by $\frac{a}{b}$ $=\mathrm{a} \cdot \mathrm{b}^{-1}$ or $\mathrm{b}^{-1} \cdot \mathrm{a}$, (they are commutative).

NOTE 3.3

In Proposition 3.2 we have seen that the equality a $\bullet \mathrm{x}=\mathrm{y}$ * $\mathrm{x}$ does not allow any kind of right-cancellation in the region A (in general, except for some particular regions). Therefore, the equality $\frac{x}{a}=\frac{x}{y}$ does not allow any kind of cancellation in the region $\mathrm{A}$; and also the equality $\frac{a}{x}=\frac{y}{x}$ does not allow any kind of cancellation in the region A (in general, except for some particular regions).

\subsubsection{Characterization of the Algebraic System "Region"}

In this section an initial characterization of region is made. Most of the useful simple results collectively all need to be validated in a unique algebraic system so that we can offer this algebraic system to the mathematicians, scientists, engineers for their use. And region is the minimal platform 
for this, neither division algebra nor any existing algebra alone. May be few results are true in division algebra, few are true in a F-algebra, few may be true in an Associative Algebra over a field F, but all the results collectively are valid in a unique algebra 'region'. That is the reason why region is unearthed and introduced with an independent and unique identity.

\section{Results 3.1}

In a region $(\mathrm{A}, \oplus, *, \bullet)$ over the field $(\mathrm{F},+,$.$) , the$ following results are therefore straightforward $\forall \mathrm{x}, \mathrm{y} \in \mathrm{A}$ and $\forall \mathrm{a} \in \mathrm{F}$ (keeping in mind that division by $0_{\mathrm{A}}$ or by $0_{\mathrm{F}}$ is not permissible):-
(i) $\mathrm{x}^{n} * \mathrm{x}^{r}=\mathrm{x}^{n+r}$
(ii) $\mathrm{x}^{n} * \mathrm{x}^{-r}=\mathrm{x}^{n-r}$
(iii) $\mathrm{x}^{-n} * \mathrm{x}^{-r}=\mathrm{x}^{-n-r}$
(iv) $(\mathrm{x} * \mathrm{y})^{\mathrm{n}}=\mathrm{x}^{\mathrm{n} *} \mathrm{y}^{\mathrm{n}}$
(v) $\left(\frac{x}{y}\right)^{n}=\frac{x^{n}}{y^{n}}$
(vi) $\left(\left(\frac{x}{a}\right)^{n}=\frac{x^{n}}{a^{n}}\right.$
(vii) $\left(\frac{a}{x}\right)^{n}=\frac{a^{n}}{x^{n}}$

where $\mathrm{n}$ and $\mathrm{r}$ are non-negative integers.

Results 3.2

The following truths in a region $\left(\mathrm{A}, \oplus,{ }^{*}, \bullet\right)$ over the field $(\mathrm{F},+,$.$) , being inherited from the definitions and properties of$ the fields $(\mathrm{A}, \oplus, *)$ and $(\mathrm{F},+,$.$) , and also of the linear Space$ $(\mathrm{A}, \oplus, \bullet)$, and of the region $\mathrm{A}$ itself, are listed below for the sake of one perusal just: -

$\forall \mathrm{x} \in \mathrm{A}$ and $\forall \mathrm{a} \in \mathrm{F}$,
(1) $0_{\mathrm{F}} \bullet \mathrm{x}=0_{\mathrm{A}}$
(2) $\mathrm{a} \bullet 0_{\mathrm{A}}=0_{\mathrm{A}}$
(3) $0_{\mathrm{F}} \bullet 0_{\mathrm{A}}=0_{\mathrm{A}}$
(4) $0_{\mathrm{F}} \bullet 0_{\mathrm{A}} \neq 0_{\mathrm{F}}$
(5) $1_{\mathrm{F}} \bullet \mathrm{x}=\mathrm{x}$
(6) $1_{\mathrm{A}} * \mathrm{x}=\mathrm{x}$
(7) $1_{\mathrm{F}} \bullet 1_{\mathrm{A}} \neq 1_{\mathrm{F}}$
(8) $1_{\mathrm{F}} \bullet 1_{\mathrm{A}}=1_{\mathrm{A}}$
(9) $\mathrm{a} \bullet 1_{\mathrm{A}} \neq \mathrm{a}$
(10) $1_{\mathrm{F}} \bullet 0_{\mathrm{A}} \neq 1_{\mathrm{F}}$
(11) $1_{\mathrm{F}} \bullet 0_{\mathrm{A}}=0_{\mathrm{A}}$
(12) $0_{\mathrm{A}} * \mathrm{X}=0_{\mathrm{A}}$
(13) $0_{\mathrm{A}} * \mathrm{X} \neq 0_{\mathrm{F}}$
(14) $1_{\mathrm{A}} \sim 1_{\mathrm{A}}=0_{\mathrm{A}}$

(15) $1_{\mathrm{F}}-1_{\mathrm{F}}=0_{\mathrm{F}}$

(16) $\frac{x}{0_{A}}$ and $\frac{x}{0_{F}}$ are meaningless.

(17) $\frac{a}{0_{A}}$ and $\frac{a}{0_{F}}$ are meaningless.

The following proposition is straightforward in any region. Proposition 3.3

In a region $\left(\mathrm{A}, \oplus,{ }^{*}, \bullet\right)$ over the field $(\mathrm{F},+,),. \forall \mathrm{x}, \mathrm{y} \in \mathrm{A}$ and $\forall \mathrm{a} \in \mathrm{F}$ (keeping in mind that division by $0_{\mathrm{A}}$ or by $0_{\mathrm{F}}$ is not permissible) the following results are true:-

(i) $\forall \mathrm{x} \in \mathrm{A}, \sim(\sim \mathrm{x})=\mathrm{x}$

(ii) $\forall \mathrm{x}\left(\neq 0_{A}\right) \in \mathrm{A},\left(\mathrm{x}^{-1}\right)^{-1}=\mathrm{x}$

(iii) $\sim(\mathrm{x} \oplus \mathrm{y})=(\sim \mathrm{x}) \oplus(\sim \mathrm{y})$

(iv) $\sim\left(\mathrm{x}^{-1}\right)=(\sim \mathrm{x})^{-1}$

(v) $(\mathrm{x} * \mathrm{y})-1=\mathrm{x}-1 * \mathrm{y}-1$

(vi) $\sim(\mathrm{x} * \mathrm{y})=(\sim \mathrm{x}) * \mathrm{y}=\mathrm{x} *(\sim \mathrm{y})$

(vii) $\sim \frac{x}{y}=\frac{\sim x}{y}=\frac{x}{\sim y}$

(viii) $\sim \frac{x}{a}=\frac{\sim x}{a}=\frac{x}{-a}$

(ix) $\sim \frac{a}{x}=\frac{-a}{x}=\frac{a}{\sim x}$

(x) $\left(\frac{x}{y}\right)^{-1}=\left(\frac{x^{-1}}{y^{-1}}\right)=\frac{y}{x}$

(xi) $\left(\frac{x}{a}\right)^{-1}=\left(\frac{x^{-1}}{a^{-1}}\right)=\frac{a}{x}$

(xii) $\left(\frac{a}{x}\right)^{-1}=\left(\frac{a^{-1}}{x^{-1}}\right)=\frac{x}{a}$

(xiii) $\mathrm{x} *(\mathrm{a} \bullet \mathrm{y})=\mathrm{a} \bullet(\mathrm{x} * \mathrm{y})$

Proof: All the results are straightforward.

Proposition 3.4

In a region $(\mathrm{A}, \oplus, *, \bullet)$ over the field $(\mathrm{F},+,),. \forall \mathrm{x} \in \mathrm{A}$, $\sim \mathrm{x}=\left(-1_{\mathrm{F}}\right) \bullet \mathrm{x}=\left(\sim 1_{\mathrm{A}}\right) * \mathrm{x}$.

Proof: We know $0_{\mathrm{A}}=0_{\mathrm{F}} \bullet \mathrm{x}$

$$
\begin{aligned}
& \text { or, } 0_{\mathrm{A}}=\left(1_{\mathrm{F}}+\left(-1_{\mathrm{F}}\right)\right) \bullet \mathrm{x} \\
& \text { or, } 0_{\mathrm{A}}=1_{\mathrm{F}} \bullet \mathrm{x} \oplus\left(-1_{\mathrm{F}}\right) \bullet \mathrm{x} \\
& \text { or, } 0_{\mathrm{A}}=\mathrm{x} \oplus\left(-1_{\mathrm{F}}\right) \bullet \mathrm{x} \\
& \text { or, }(\sim \mathrm{x}) \oplus 0_{\mathrm{A}}=(\sim \mathrm{x}) \oplus\left(\mathrm{x} \oplus\left(-1_{\mathrm{F}}\right) \bullet \mathrm{x}\right) \\
& \text { or, } \sim \mathrm{x}=\left(-1_{\mathrm{F}}\right) \bullet \mathrm{x} \text { which is the result. }
\end{aligned}
$$

Again, we have, $0_{\mathrm{A}}=0_{\mathrm{A}} * \mathrm{x}$

$$
\begin{aligned}
& \text { or, } 0_{\mathrm{A}}=\left(1_{\mathrm{A}} \oplus\left(\sim 1_{\mathrm{A}}\right)\right) * \mathrm{x} \\
& \text { or, } 0_{\mathrm{A}}=1_{\mathrm{A}} * \mathrm{x} \oplus\left(\sim 1_{\mathrm{A}}\right) * \mathrm{x} \\
& \text { or, } 0_{\mathrm{A}}=\mathrm{x} \oplus\left(\sim 1_{\mathrm{A}}\right) * \mathrm{x} \\
& \text { or, } \sim \mathrm{x} \oplus 0_{\mathrm{A}}=\sim \mathrm{x} \oplus\left(\mathrm{x} \oplus\left(\sim 1_{\mathrm{A}}\right) * \mathrm{x}\right) \\
& \text { or, } \sim \mathrm{x}=\left(\sim 1_{\mathrm{A}}\right) * \mathrm{x}, \text { which is the result. }
\end{aligned}
$$

Proposition 3.5

In an infinite region $\mathrm{A}=(\mathrm{A}, \oplus, *, \bullet)$ over the field $(\mathrm{F}$, ,+ .) where the characteristic of $\mathrm{A}$ is zero, if $\mathrm{a} \bullet \mathrm{x}=0_{\mathrm{A}}$ then either $\mathrm{a}=0_{\mathrm{F}}$ or $\mathrm{x}=0_{\mathrm{A}}$, where $\mathrm{x} \in \mathrm{A}$ and $\mathrm{a} \in \mathrm{F}$.

Proof: We have $\mathrm{a} \bullet \mathrm{x}=0_{\mathrm{A}}$. 


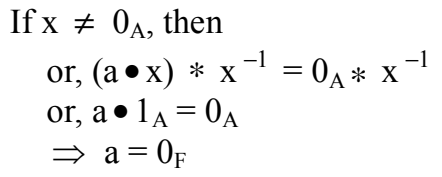

Otherwise, if $\mathrm{a} \neq 0_{\mathrm{F}}$, then

$$
\begin{aligned}
& \mathrm{a}^{-1} \bullet(\mathrm{a} \bullet \mathrm{x})=\mathrm{a}^{-1} \bullet 0_{\mathrm{A}} \\
& \text { or, }\left(\mathrm{a}^{-1} \cdot \mathrm{a}\right) \bullet \mathrm{x}=0_{\mathrm{A}} \\
& \text { or, } 1_{\mathrm{F}} \bullet \mathrm{x}=0_{\mathrm{A}} \\
& \text { or, } \mathrm{x}=0_{\mathrm{A}} \text {. Hence the result. }
\end{aligned}
$$

In a region $(\mathrm{A}, \oplus, *, \bullet)$ over the field $(\mathrm{F},+,$.$) , we know that$ $\forall \mathrm{a} \in \mathrm{F}$ and $\forall \mathrm{x} \in \mathrm{A}$, the element $(\mathrm{a} \bullet \mathrm{x})$ is in A. Therefore, $(\mathrm{a} \bullet \mathrm{x})$ possesses its additive inverse in the region A. Also, if it is not the zero-element of the region $\mathrm{A}$ then it possesses its multiplicative inverse too in the region $\mathrm{A}$. The following proposition defines the additive inverse of the element $(\mathrm{a} \bullet \mathrm{x})$ in the region $\mathrm{A}$.

\section{Proposition 3.6}

In a region $(\mathrm{A}, \oplus, *, \bullet)$ over the field $(\mathrm{F},+,),. \forall \mathrm{x} \in \mathrm{A}$ and $\forall \mathrm{a} \in \mathrm{F}, \sim(\mathrm{a} \bullet \mathrm{x})=(-\mathrm{a}) \bullet \mathrm{x}=\mathrm{a} \bullet(\sim \mathrm{x})$.

Proof: $(\mathrm{a} \bullet \mathrm{x}) \oplus((-\mathrm{a}) \bullet \mathrm{x})$

$$
\begin{aligned}
& =(\mathrm{a}+(-\mathrm{a})) \bullet \mathrm{x} \\
& =0_{\mathrm{F}} \bullet \mathrm{x} \\
& =0_{\mathrm{A}} \\
& \therefore \sim(\mathrm{a} \bullet \mathrm{x})=(-\mathrm{a}) \bullet \mathrm{x}
\end{aligned}
$$

In a similar way, we can also prove that $\sim(\mathrm{a} \bullet \mathrm{x})=\mathrm{a} \bullet(\sim \mathrm{x})$.

The following proposition defines the multiplicative inverse of the element $(\mathrm{a} \bullet \mathrm{x})$ in the region $\mathrm{A}$. This important result is not valid in a division algebra, in general.

Proposition 3.7

In a region $(\mathrm{A}, \oplus, *, \bullet)$ over the field $(\mathrm{F},+,),. \forall \mathrm{x}\left(\neq 0_{\mathrm{A}}\right)$ $\in \mathrm{A}$ and $\forall \mathrm{a}\left(\neq 0_{\mathrm{F}}\right) \in \mathrm{F},(\mathrm{a} \bullet \mathrm{x})^{-1}=\mathrm{a}^{-1} \bullet \mathrm{x}^{-1}$.

Proof: $(\mathrm{a} \bullet \mathrm{x}) *\left(\mathrm{a}^{-1} \bullet \mathrm{x}^{-1}\right)$

$$
\begin{aligned}
& =\left(\mathrm{a}^{-1}\right) \bullet\left(\mathrm{x} * \mathrm{x}^{-1}\right), \text { using compatibility property of } \\
& \quad \text { region A. } \\
& =1_{\mathrm{F}} \bullet 1_{\mathrm{A}} \\
& =1_{\mathrm{A}}
\end{aligned}
$$

Therefore, $(\mathrm{a} \bullet \mathrm{x})^{-1}=\mathrm{a}^{-1} \bullet \mathrm{x}^{-1}$.

This following important result is not valid in a division algebra, in general.

Proposition 3.8

In a region $(\mathrm{A}, \oplus, *, \bullet)$ over the field $(\mathrm{F},+,$.$) , for \mathrm{x}, \mathrm{y}$ $\rho_{\text {new }} \mathrm{A}$ and for $\mathrm{a}, \mathrm{b} \in \mathrm{F}$, if $\mathrm{b}$ and $\mathrm{y}$ are not zero elements then

$$
\frac{a}{b} \bullet \frac{x}{y}=\frac{a \bullet x}{b \bullet y}
$$

$$
\text { Proof: } \begin{aligned}
\frac{a}{b} \bullet \frac{x}{y} & =\left(\mathrm{a} \cdot \mathrm{b}^{-1}\right) \bullet\left(\mathrm{x}^{*} \mathrm{y}^{-1}\right) \\
& =(\mathrm{a} \bullet \mathrm{x})^{*}\left(\mathrm{~b}^{-1} \bullet \mathrm{y}^{-1}\right) \\
& =(\mathrm{a} \bullet \mathrm{x})^{*}(\mathrm{~b} \bullet \mathrm{y})^{-1} \\
& =\frac{a \bullet x}{b \bullet y}
\end{aligned}
$$

The results of the following proposition can be established easily in a region.

\section{Proposition 3.9}

In a region $\left(\mathrm{A}, \oplus,{ }^{*}, \bullet\right)$ over the field $(\mathrm{F},+,),. \forall \mathrm{x}, \mathrm{y} \in \mathrm{A}$ and $\forall \mathrm{a}, \mathrm{b} \in \mathrm{F}$ (keeping in mind that a zero element have its inverse),

(i) $(\mathrm{a} \bullet(\mathrm{x} * \mathrm{y}))^{-1}=\mathrm{a}^{-1} \bullet \mathrm{x}^{-1} * \mathrm{y}^{-1}$

(ii) $\sim(\mathrm{a} \bullet(\mathrm{x} * \mathrm{y}))=(-\mathrm{a}) \bullet(\mathrm{x} * \mathrm{y})=\mathrm{a} \rho_{\text {new }}(x, y)((\sim \mathrm{x}) *$ $\mathrm{y})=\mathrm{a} \bullet(\mathrm{x} *(\sim \mathrm{y}))$

(iii) $\mathrm{a} \bullet \mathrm{x} * \mathrm{~b} \bullet \mathrm{y})^{-1}=\left(\mathrm{a}^{-1} \bullet \mathrm{x}^{-1}\right) *\left(\mathrm{~b}^{-1} \bullet \mathrm{y}^{-1}\right)$

(iv) $\sim(\mathrm{a} \bullet \mathrm{x} * \mathrm{~b} \bullet \mathrm{y})=(\sim(\mathrm{a} \bullet \mathrm{x})) *(\mathrm{~b} \bullet \mathrm{y})=(\mathrm{a} \bullet \mathrm{x}) *(\sim(\mathrm{b} \bullet$ $\mathrm{y}))$

(v) $((a \cdot b) \bullet x)^{-1}=\left(a^{-1} \cdot b^{-1}\right) \bullet x^{-1}$

(vi) $\sim((a . b) \bullet x)=((-a) . b) \bullet x=(a .(-b)) \bullet x=(a . b)$ $(\sim \mathrm{x})$

Many of the frequently practiced cancellation laws are not valid in a Division Algebra alone or in any existing standard algebraic system alone, by their definitions or by virtue of their respective properties.

For example, the result $\frac{a \bullet x}{a \bullet y}=\frac{x}{y}$ is not valid in a Division Algebra alone or in any of the existing standard algebraic systems alone.

The following valid cancellation laws ensures the superiority of region over any existing algebraic system as a single brand.

\section{Proposition 3.10 Cancellation Laws}

Let $\left(\mathrm{A}, \oplus,{ }^{*}, \bullet\right)$ be a region over the field $(\mathrm{F},+$, .). Since $(\mathrm{A}$, $\left.\oplus,{ }^{*}\right)$ is a field, the following cancellation laws hold good in a region $(\mathrm{A}, \oplus, *, \bullet)$ by virtue of inheritance:-

(1) If $\mathrm{x} \oplus \mathrm{y}=\mathrm{x} \oplus \mathrm{z}$, then $\mathrm{y}=\mathrm{z}$ where $\mathrm{x}, \mathrm{y}, \mathrm{z} \in A$.

(2) If $x \oplus y=z \oplus y$, then $x=z$ where $x, y, z \in A$.

(3) If $x * y=x * z$ where $x \neq 0_{A}$, then $y=z$ where $x, y, z \in$ A.

(4) If $x * y=z * y$ where $y \neq 0$, then $x=z$ where $x, y, z \in$ A.

However, it can be easily shown that the following two cancellation laws too hold good in a region A:-

(5) If $a \bullet x=a \bullet y$ where $a \neq 0$ F, then $x=y$ where $x, y \in$ $A$ and $\mathrm{a} \in \mathrm{F}$.

(6) If $\mathrm{a} \bullet \mathrm{x}=\mathrm{b} \bullet \mathrm{x}$ where $\mathrm{x} \neq 0_{\mathrm{A}}$, then $\mathrm{a}=\mathrm{b}$ where $\mathrm{x} \in \mathrm{A}$ and $a, b \in F$.

Besides the above six, there are a number of kinds of cancellation operations valid in the region $\left(\mathrm{A}, \oplus,{ }^{*}, \bullet\right)$ over the field $(\mathrm{F},+,$.$) , few of which are quoted below:-$

If $x, y \in A$ and $a, b \in F$, then

(7) If (a.b) $\bullet \mathrm{x}=($ a.c $) \bullet \mathrm{y}$ where $\mathrm{a} \neq 0$ F, then $\mathrm{b} \bullet \mathrm{x}=\mathrm{c} \bullet \mathrm{y}$.

(8) $\frac{a \bullet x}{a \bullet y}=\frac{x}{y}$, where $\mathrm{a} \neq 0_{\mathrm{F}}$ and $\mathrm{y} \neq 0_{\mathrm{A}}$.

(9) $\frac{(a \cdot b) \bullet x}{(a \cdot c) \bullet y}=\frac{b \bullet x}{c \bullet y}$, where $\mathrm{a} \neq 0_{\mathrm{F}}$.

(10) $\frac{(a \cdot c) \bullet x}{(b \cdot c) \bullet y}=\frac{a \bullet x}{b \bullet y}$, where $\mathrm{c} \neq 0_{\mathrm{F}}$.

(11) $\frac{(a \bullet x) * y}{(b \bullet x) * z}=\frac{a \bullet y}{b \bullet z}$, where $\mathrm{x} \neq 0_{\mathrm{A}}$. 
(12) $\frac{(a \bullet x) * y}{(a \bullet z) * t}=\frac{x * y}{z * t}$, where $\mathrm{a} \neq 0_{\mathrm{F}}$

Proof: Although the proof of all the above results are straightforward, nevertheless we present below proof of one result (8).

$$
\begin{gathered}
\frac{a \bullet x}{a \bullet y}=(\mathrm{a} \bullet \mathrm{x}) *(\mathrm{a} \bullet \mathrm{y})^{-1} \\
=(\mathrm{a} \bullet \mathrm{x}) *\left(\mathrm{a}^{-1} \bullet \mathrm{y}^{-1}\right)
\end{gathered}
$$

$=\left(\mathrm{a} \cdot \mathrm{a}^{-1}\right)^{\bullet}\left(\mathrm{x}^{*} \mathrm{y}^{-1}\right)$, using compatibility property of region A.

$$
\begin{gathered}
=1_{\mathrm{F}} \bullet\left(\mathrm{x}^{*} \mathrm{y}^{-1}\right) \\
=\mathrm{x}^{*} \mathrm{y}^{-1} \\
=\frac{x}{y}
\end{gathered}
$$

\section{NOTE 3.4}

Proposition 3.2 states that in a region $\left(\mathrm{A}, \oplus,{ }^{*}, \bullet\right)$ over the field $(\mathrm{F},+,$.$) , for \mathrm{a} \in \mathrm{F}$ and for $\mathrm{x}\left(\neq 0_{\mathrm{A}}\right), \mathrm{y} \in \operatorname{region} \mathrm{A}$, if $\mathrm{y} *$ $\mathrm{x}=\mathrm{a} \bullet \mathrm{x}$ then $\mathrm{y}=\mathrm{a} \bullet 1_{\mathrm{A}}$, and thus there is no kind of right cancellation holds good here.

Proposition 3.11

In a region $(\mathrm{A}, \oplus, *, \bullet)$ over the field $(\mathrm{F},+,$.$) , the following$ results are true (keeping in mind that division by $0_{\mathrm{A}}$ or $0_{\mathrm{F}}$ is not permissible):-

If $\mathrm{x}, \mathrm{y}, \mathrm{z}, \mathrm{t} \in \mathrm{A}$, and $\mathrm{a}, \mathrm{b}, \mathrm{c}, \mathrm{d} \in \mathrm{F}$, then

(i) $\frac{x}{y} * \frac{z}{t}=\frac{x * z}{y * t}$

(ii) $\frac{x \oplus y}{z}=\left(\frac{x}{z}\right) \oplus\left(\frac{y}{z}\right)$

(iii) $\mathrm{x}^{2} \sim \mathrm{y}^{2}=(\mathrm{x} \oplus \mathrm{y}) *(\mathrm{x} \sim \mathrm{y})$

(iv) $\frac{x}{y} \oplus \frac{z}{t}=\frac{(x * t) \oplus(y * z)}{y * t}$

(v) $\frac{a \bullet x \oplus b \bullet y}{c \bullet z}=\left(\frac{a \bullet x}{c \bullet z}\right) \oplus\left(\frac{b \bullet y}{c \bullet z}\right)=\left(\frac{a}{c}\right) \bullet\left(\frac{x}{z}\right) \oplus\left(\frac{b}{c}\right) \bullet\left(\frac{y}{z}\right)$

Proof: All the results are straightforward, nevertheless we present the proof of result-(iv) here.

$$
\begin{aligned}
& \frac{x}{y} \oplus \frac{z}{t} \\
= & \left(\mathrm{x} * \mathrm{y}^{-1)} \oplus\left(\mathrm{z}^{*} \mathrm{t}^{-1}\right)\right. \\
= & \left((\mathrm{x} * \mathrm{t}) *\left(\mathrm{y}^{-1} * \mathrm{t}^{-1}\right)\right) \oplus\left((\mathrm{y} * \mathrm{z}) *\left(\mathrm{y}^{-1} * \mathrm{t}^{-1}\right)\right) \\
= & ((\mathrm{x} * \mathrm{t}) \oplus(\mathrm{y} * \mathrm{z})) *\left(\mathrm{y}^{-1} * \mathrm{t}^{-1}\right) \\
= & ((\mathrm{x} * \mathrm{t}) \oplus(\mathrm{y} * \mathrm{z})) *(\mathrm{y} * \mathrm{t})^{-1} \\
= & \frac{x * t \oplus y * z}{y * t}
\end{aligned}
$$

The results of the following proposition is also straightforward in any region, but not true in a Division
Algebra in general.

Proposition 3.12

In a region $(\mathrm{A}, \oplus, *, \bullet)$ over the field $(\mathrm{F},+,$.$) , for any$ non-negative integer $n$, if $x \in A$ and $a, b \in F$ then the following results are true.

(i) $(\mathrm{a} \bullet \mathrm{x})^{n}=\mathrm{a}^{n} \bullet \mathrm{x}^{n}$ (ii) $\left(\frac{a \bullet x}{b \bullet y}\right)^{n}=\frac{a^{n} \cdot x^{n}}{b^{n} \cdot y^{n}}=\frac{a^{n}}{b^{n}} \cdot \frac{x^{n}}{y^{n}}$

where $\mathrm{b} \neq 0_{\mathrm{F}}$ and $\mathrm{y} \neq 0_{\mathrm{A}}$.

NOTE 3.5

The equality $\mathrm{a} \bullet \mathrm{x}=\mathrm{b} \bullet \mathrm{y}$ implies the following equalities (keeping in mind that division by $0_{\mathrm{A}}$ or by $0_{\mathrm{F}}$ is not permissible):

(i) $\frac{1_{F}}{b} \bullet \mathrm{x}=\frac{1_{F}}{a} \bullet \mathrm{y}$.

(ii) $\mathrm{a} \bullet \frac{1_{A}}{y}=\mathrm{b} \bullet \frac{1_{A}}{x}$.

(iii) $\frac{x}{b}=\frac{y}{a}$

(iv) $\frac{a}{y}=\frac{b}{x}$.

But the equality $\mathrm{a} \bullet \mathrm{x}=\mathrm{b} \bullet \mathrm{y}$ can not imply that $\frac{a}{b}=\frac{y}{x}$. In fact this is an invalid and absurd equality, although both $\frac{a}{b}$ and $\frac{y}{x}$ are individually meaningful. However, it surely implies the following equalities:-

(i) $\frac{y}{x}=\frac{a}{b} \bullet 1_{\mathrm{A}}$ and (ii) $\frac{x}{y}=\frac{b}{a} \bullet 1_{\mathrm{A}}$

One of the most useful and most important properties fluently applied by the mathematicians in their calculations is Cross Multiplication Property. The following simple Cross Multiplication Property is not valid in general in a division algebra alone or in any existing standard algebraic system alone, by their respective definition or by virtue of their respective properties. But the same result is well valid in a region $\mathrm{A}$.

Proposition 3.13 Cross Multiplication Property

In a region $\left(\mathrm{A}, \oplus,{ }^{*}, \bullet\right)$ over the field $(\mathrm{F},+,$.$) , the Cross$ Multiplication Property is well valid. i.e.

If $\frac{a \bullet x}{b \bullet y}=\frac{c \bullet z}{d \bullet t}$, then (a.d) $\bullet(\mathrm{x} * \mathrm{t})=(\mathrm{b} . \mathrm{c}) \bullet(\mathrm{y} * \mathrm{z})$ and conversely,

where $\mathrm{x}, \mathrm{y}, \mathrm{z}, \mathrm{t} \in \mathrm{A}, \mathrm{y} \neq 0_{\mathrm{A}} \neq \mathrm{t}$ and $\mathrm{a}, \mathrm{b}, \mathrm{c}, \mathrm{d} \in \mathrm{F}, \mathrm{b} \neq 0_{\mathrm{F}} \neq \mathrm{d}$.

Proof: We have $\frac{a \bullet x}{b \bullet y}=\frac{c \bullet z}{d \bullet t}$

$$
\begin{aligned}
& \text { i.e. }(\mathrm{a} \bullet \mathrm{x}) *(\mathrm{~b} \bullet \mathrm{y})^{-1}=(\mathrm{c} \bullet \mathrm{z}) *(\mathrm{~d} \bullet \mathrm{t})^{-1} \\
& \text { or, }(\mathrm{a} \bullet \mathrm{x}) *(\mathrm{~d} \bullet \mathrm{t})=(\mathrm{b} \bullet \mathrm{y}) *(\mathrm{c} \bullet \mathrm{z}) \\
& \text { or, }(\mathrm{a} . \mathrm{d}) \bullet(\mathrm{x} * \mathrm{t})=(\mathrm{b} . \mathrm{c}) \bullet(\mathrm{y} * \mathrm{z}) .
\end{aligned}
$$

Proposition 3.14

In a region $\left(\mathrm{A}, \oplus,{ }^{*}, \bullet\right)$ over the field $(\mathrm{F},+,$.$) , if \mathrm{a} \bullet \mathrm{x}=\mathrm{b} \bullet \mathrm{y}$ 
then $\frac{a \bullet x}{b \bullet y}=1_{\mathrm{A}}$ and conversely,

where $x, y \in A, a, b \in F$ and $b \bullet y \neq 0$.

The so important result (result-(ii) below) of Componendo \& Dividendo Rule is not valid in a Division Algebra alone or in any existing standard algebraic system alone, by their respective definitions or by virtue of their respective properties. But the same result is well valid in a region A (i.e. the minimum platform is a region).

Proposition 3.15 Componendo \& Dividendo Rule

In a region $\left(\mathrm{A}, \oplus,{ }^{*}, \bullet\right)$ over the field $(\mathrm{F},+,$.$) , the following$ 'Componendo \& Dividendo' rules are well valid:

(i) If $\frac{x}{y}=\frac{z}{t}$, then $\frac{x}{y}=\frac{z}{t}=\frac{x \oplus z}{y \oplus t}=\frac{x \sim z}{y \sim t}$, where $\mathrm{x}$, $\mathrm{y}, \mathrm{z}, \mathrm{t} \in \mathrm{A}$, and denominator $\neq 0_{\mathrm{A}}$.

(ii) If $\frac{x}{y}=\frac{z}{t}$, then $\frac{x}{y}=\frac{z}{t}=\frac{(a \bullet x) \oplus(b \bullet z)}{(a \bullet y) \oplus(b \bullet t)}=$ $\frac{a \bullet x \sim b \bullet z}{a \bullet y \sim b \bullet t}$

where $\mathrm{x}, \mathrm{y}, \mathrm{z}, \mathrm{t} \in \mathrm{A}$, and denominator $\neq 0_{\mathrm{A}}$.

Proof:

(i) We have $\frac{x}{y}=\frac{z}{t}$

or, $\mathrm{x} * \mathrm{y}^{-1}=\mathrm{z} * \mathrm{t}^{-1}$

or, $\left(\mathrm{x} * \mathrm{y}^{-1}\right) *(\mathrm{y} * \mathrm{t})=\left(\mathrm{z} * \mathrm{t}^{-1}\right) *(\mathrm{y} * \mathrm{t})$

or, $\mathrm{x} * \mathrm{t}=\mathrm{z} * \mathrm{y}$

or, $\mathrm{x} * \mathrm{y} \oplus \mathrm{x} * \mathrm{t}=\mathrm{x} * \mathrm{y} \oplus \mathrm{z} * \mathrm{y}$

or, $\mathrm{x} *(\mathrm{y} \oplus \mathrm{t})=(\mathrm{x} \oplus \mathrm{z}) * \mathrm{y}$

or, $\frac{x}{y}=\frac{x \oplus z}{y \oplus t}$

In a similar way we can establish that $\frac{x}{y}=\frac{x \sim z}{y \sim t}$. Hence the result.

(ii) We have $\frac{x}{y}=\frac{z}{t}$

$$
\text { Now, } \begin{aligned}
\frac{x}{y} & =\mathrm{x}^{*} \mathrm{y}^{-1} \\
& =\left(\mathrm{a} \cdot \mathrm{a}^{-1}\right) \bullet\left(\mathrm{x} * \mathrm{y}^{-1}\right), \bullet \\
& =(\mathrm{a} \bullet \mathrm{x}) *\left(\mathrm{a}^{-1} \bullet \mathrm{y}^{-1}\right), \text { using compatibility } \\
& \text { property of region A. } \\
= & (\mathrm{a} \bullet \mathrm{x}) *\left(\mathrm{a} \bullet \mathrm{y}^{-1}\right. \\
= & \frac{a \bullet x}{a \bullet y}
\end{aligned}
$$

Similarly, we can also establish that $\frac{z}{t}=\frac{b \bullet z}{b \bullet t}$.

Now, we have

$$
\frac{x}{y}=\frac{a \bullet x}{a \bullet y}=\frac{b \bullet z}{b \bullet t}=\frac{z}{t}
$$

Applying now the result (i), we have

$$
\frac{x}{y}=\frac{a \bullet x \oplus b \bullet z}{a \bullet y \oplus b \bullet t}=\frac{a \bullet x \sim b \bullet z}{a \bullet y \sim b \bullet t}=\frac{z}{t} .
$$

\subsubsection{Characteristic of a Region}

In Region Algebra, the characteristic of a region A denoted char(A) is defined to be the smallest number of times one must use its multiplicative identity $1_{\mathrm{A}}$ in a sum to get the additive identity element $0_{\mathrm{A}}$. A region is said to have characteristic zero if this sum never reaches the additive identity. For example, char(RR) $=0$.

\subsection{Categories of Regions}

In this section three special types of regions are discussed which are useful to the mathematicians. These regions will be applicable in the subsequent sections here in our course of introducing "Region Mathematics".

\subsubsection{Real Region}

A region $\left(\mathrm{A}, \oplus,{ }^{*}, \bullet\right)$ over the field $(\mathrm{F},+,$.$) is called a Real$ Region if its outer field $\mathrm{F}$ is the classical field $\mathrm{R}$ of real numbers.

\section{Example 3.5}

The region RR (see earlier Example 3.4), C are examples of real region.

The following simple results/formulas (Proposition $3.16,3.17,3.18)$ are very useful and important results valid in regions, but all these collectively are not valid in general in division algebra alone or in any of the existing classical algebraic systems alone by their respective definitions and by virtue of their respective properties. These results reduce to the corresponding important classical results of elementary algebra as special cases. This is in fact the major applications of the regions in mathematics/algebra. The main philosophy behind this work is to discover that particular algebraic system in which such type of simple but useful results, equalities, solutions, formulas etc. of elementary algebra are valid (can be computed and verified).

Proposition 3.16

The following results hold good in a real region $(\mathrm{A}, \oplus, *$, $\bullet)$ :

(i) $\left(1_{\mathrm{A}} \oplus \mathrm{x}\right)^{2}=1_{\mathrm{A}} \oplus 2 \bullet \mathrm{x} \oplus \mathrm{x}^{2}, \forall \mathrm{x} \in \mathrm{A}$

(ii) $(\mathrm{x} \oplus \mathrm{y})^{2}=\mathrm{x}^{2} \oplus 2 \bullet \mathrm{x} * \mathrm{y} \oplus \mathrm{y}^{2}, \forall \mathrm{x}, \mathrm{y} \in \mathrm{A}$

(iii) $(\mathrm{x} \sim \mathrm{y})^{2}=\mathrm{x}^{2} \sim 2 \bullet \mathrm{x} * \mathrm{y} \oplus \mathrm{y}^{2}, \forall \mathrm{x}, \mathrm{y} \in \mathrm{A}$

(iv) $(\mathrm{x} \oplus \mathrm{y})^{3}=\mathrm{x}^{3} \oplus 3 \bullet \mathrm{x}^{2} * \mathrm{y} \oplus 3 \bullet \mathrm{x} * \mathrm{y}^{2} \oplus \mathrm{y}^{3}, \forall \mathrm{x}, \mathrm{y} \in \mathrm{A}$

(v) $(\mathrm{x} \sim \mathrm{y})^{3}=\mathrm{x}^{3} \sim 3 \bullet \mathrm{x}^{2 *} \mathrm{y} \oplus 3 \bullet \mathrm{x} * \mathrm{y}^{2} \sim \mathrm{y}^{3}, \forall \mathrm{x}, \mathrm{y} \in \mathrm{A}$

However, the above results in general are not true in a region which is not a real region. A generalized result is given below.

Proposition 3.17

In a real region $(\mathrm{A}, \oplus, *, \bullet)$ the following equality is valid $\forall \mathrm{x}, \mathrm{y} \in \mathrm{A}$ :

$$
(\mathrm{x} \oplus \mathrm{y})^{\mathrm{n}}=\sum_{r=0}^{n}\left(\begin{array}{l}
n \\
r
\end{array}\right) \bullet\left(\mathrm{x}^{\mathrm{n}-\mathrm{r} *} \mathrm{y}^{\mathrm{r}}\right)
$$

where the notation $\sum$ stands for summation over the 
symbol $\oplus$ of first addition of the region $A$, and $n$ is a positive integer.

(However, this result in general is not true in a region if it is not a real region).

NOTE 3.6

The RR region is the actual algebraic system in which most of the results, expressions, equalities of school algebra are studied and taught. In RR region, the results of Proposition $3.16 \& 3.17$ are being written by all in traditional style as below:-
(i) $(1+x)^{2}=1+2 x+x^{2}$
(ii) $(x+y)^{2}=x^{2}+2 x y+y^{2}$
(iii) $(x-y)^{2}=x^{2}-2 x y+y^{2}$
(iv) $(x+y)^{3}=x^{3}+3 x^{2} y+3 x y^{2}+y^{3}$
(v) $(x-y)^{3}=x^{3}-3 x^{2} y+3 x y^{2}-y^{3}$
(vi) $(\mathrm{x}+\mathrm{y})^{\mathrm{n}}=\sum_{r=0}^{n}\left(\begin{array}{l}n \\ r\end{array}\right) \mathrm{x}^{\mathrm{n}-\mathrm{r}} \mathrm{y}^{\mathrm{r}}$

The following results are not valid in a division algebra alone or in any of the existing classical algebraic systems alone by their respective definitions and by virtue of their respective properties, but valid in any algebraic system which is at least a real region.

Proposition 3.18

If $(\mathrm{A}, \oplus, *, \bullet)$ be a real region, then the following results are true $\forall \mathrm{x}, \mathrm{y} \in \mathrm{A}$ and $\forall \mathrm{a}, \mathrm{b} \in \mathrm{R}$ :

(i) $(\mathrm{a} \bullet \mathrm{x} \oplus \mathrm{b} \bullet \mathrm{y}) 2=\mathrm{a}^{2} \bullet \mathrm{x}^{2} \oplus \mathrm{b}^{2} \bullet \mathrm{y}^{2} \quad(2 . \mathrm{a} . \mathrm{b}) \bullet(\mathrm{x} *$ $\mathrm{y})$.

(ii) $(\mathrm{a} \bullet \mathrm{x} \oplus \mathrm{b} \bullet \mathrm{y})^{n}=\sum_{r=0}^{n}\left(\left(\begin{array}{l}n \\ r\end{array}\right) a^{n-r} \cdot b^{r}\right) \bullet\left(x^{n-r} * y^{r}\right)$.

NOTE 3.7

However, in RR region the above results are written by all in traditional style as below:-

(i) $(a x+b y)^{2}=a^{2} x^{2}+b^{2} y^{2}+2 a b x y$

(ii) $(\mathrm{ax}+\mathrm{by})^{n}=\sum_{r=0}^{n}\left(\left(\begin{array}{l}n \\ r\end{array}\right) a^{n-r} b^{r} x^{n-r} y^{r}\right)$

\subsubsection{Region over a Region (ROR)}

Let $(\mathrm{A}, \oplus, *, \bullet)$ be a region over a field $(\mathrm{F},+,$.$) . If the$ algebraic system $(\mathrm{F},+, . .$.$) itself be a region over a field (\mathrm{K}$, \pm .), then we say that A is a 'Region over a Region' (or, ROR). In such case the region $F$ is called the 'base region' of the ROR A.

\subsubsection{Region over a Real Region (RORR)}

If the base region is a real region, then $A$ is called a 'Region over a Real Region' (or, RORR). An example of RORR is the region $\mathrm{RR}$.

Proposition 3.19

If $\mathrm{A}=(\mathrm{A}, \oplus, *, \bullet)$ is a region over a real region $\mathrm{F}$, then $\forall \mathrm{x} \in \mathrm{A}$
(i) $\mathrm{x} \oplus \mathrm{x}=\left(2.1_{\mathrm{F}}\right) \bullet \mathrm{x}$
(ii) $\sum_{r=1}^{n} \mathrm{x}=\left(\right.$ n. $\left.1_{\mathrm{F}}\right) \bullet \mathrm{x}$

$$
\text { Proof: } \begin{aligned}
\mathrm{x} \oplus \mathrm{x} & =\left(1_{\mathrm{F}} \bullet \mathrm{x}\right) \oplus\left(1_{\mathrm{F}} \bullet \mathrm{x}\right) \\
& =\left(1_{\mathrm{F}}+1_{\mathrm{F}}\right) \bullet \mathrm{x} \\
& =\left(1.1_{\mathrm{F}}+1.1_{\mathrm{F}}\right) \bullet \mathrm{x} \\
& =\left(2.1_{\mathrm{F}}\right) \bullet \mathrm{x} \text { Hence the result. }
\end{aligned}
$$

The result (ii) can be proved similarly.

Proposition 3.20

If $\mathrm{A}=(\mathrm{A}, \oplus, *, \bullet)$ is a region over a real region $\mathrm{F}$, then $\forall$ $\mathrm{x}, \mathrm{y} \in \mathrm{A}$

(i) $\left(1_{\mathrm{A}} \oplus \mathrm{x}\right) 2=1_{\mathrm{A}} \oplus\left(2.1_{\mathrm{F}}\right) \bullet \mathrm{x} \oplus \mathrm{x} 2$

(ii) $(\mathrm{x} \oplus \mathrm{y}) 2=\mathrm{x} 2 \oplus\left(2.1_{\mathrm{F}}\right) \bullet \mathrm{x} * \mathrm{y} \oplus \mathrm{y} 2$

(iii) $\left(\mathrm{x}^{\oplus} \mathrm{y}\right)^{n}=\sum_{r=0}^{n}\left(\left(\begin{array}{l}n \\ r\end{array}\right) \cdot 1_{F}\right) \cdot x^{n-r} * y^{r} \quad$ where $\mathrm{x}^{0}=1_{\mathrm{A}}$ and $\mathrm{a}^{0}=1_{\mathrm{F}}$.

(iv) $(\mathrm{a} \bullet \mathrm{x} \oplus \mathrm{b} \bullet \mathrm{y}) 2=\mathrm{a}^{2} \bullet \mathrm{x}^{2} \oplus \mathrm{b}^{2} \bullet \mathrm{y}^{2}(2 .(\mathrm{a} . \mathrm{b})) \bullet(\mathrm{x} * \mathrm{y})$

(v) $(\mathrm{a} \bullet \mathrm{x} \oplus \mathrm{b} \bullet \mathrm{y})^{n}=\sum_{r=0}^{n}\left(\left(\begin{array}{l}n \\ r\end{array}\right) \cdot a^{n-r} \cdot b^{r}\right) \bullet\left(x^{n-r} * y^{r}\right)$

The following results are straightforward.

Proposition 3.21

If the region $(\mathrm{A}, \oplus, *, \bullet)$ is a real region, then $\forall \mathrm{x}, \mathrm{y} \in \mathrm{A}$ the following results are true (not necessarily true in general if the region $\mathrm{A}$ is not a real region):-

(i) $\mathrm{x}^{2} \oplus \mathrm{y}^{2}=(\mathrm{x} \oplus \mathrm{y})^{2} \sim 2 .(\mathrm{x} * \mathrm{y})=(\mathrm{x} \sim \mathrm{y})^{2} \oplus 2 .(\mathrm{x} *$ y)

(ii) $(\mathrm{x} \sim \mathrm{y})^{2}=(\mathrm{x} \oplus \mathrm{y})^{2} \sim 4$. $(\mathrm{x} * \mathrm{y})$

(iii) $(\mathrm{x} \oplus \mathrm{y})^{2}=(\mathrm{x} \sim \mathrm{y})^{2} \oplus 4 .(\mathrm{x} * \mathrm{y})$

(iv) $\mathrm{x}^{3} \sim \mathrm{y}^{3}=(\mathrm{x} \sim \mathrm{y}) *\left(\mathrm{x}^{2} \oplus \mathrm{x} * \mathrm{y} \oplus \mathrm{y}^{2}\right)=(\mathrm{x} \sim \mathrm{y})^{3} \oplus 3$. $((\mathrm{x} * \mathrm{y}) *(\mathrm{x} \sim \mathrm{y}))$

(v) $\mathrm{x}^{3} \oplus \mathrm{y}^{3}=(\mathrm{x} \oplus \mathrm{y}) *\left(\mathrm{x}^{2} \sim \mathrm{x} * \mathrm{y} \oplus \mathrm{y}^{2}\right)=(\mathrm{x} \oplus \mathrm{y})^{3}$ $\sim 3 .((\mathrm{x} * \mathrm{y}) *(\mathrm{x} \oplus \mathrm{y}))$

There are many (most of the) algebraic problems at secondary school level of 'elementary algebra' which we solve without knowing the identity of the minimal algebraic system upon which we are having our right to solve them. For example, the following problem is a very simple problem of school level 'elementary algebra' which can not be solved in general in groups alone, or in rings alone, or in modules, fields, module, linear spaces, algebra over a field, associative algebra over a field, division algebra alone or in any existing classical algebraic system alone, by their respective definitions and by virtue of their respective properties, but can only be solved in a region or in an algebraic system which is at least a region. This breakthrough is justified here in the immediate Note below.

Problem 3.1.

Solve for $\mathrm{x}$ the equation: $3^{\bullet} \mathrm{x} * \mathrm{y}=2 \bullet \mathrm{y} \oplus 3^{\bullet} \mathrm{t}$, where $\mathrm{x}, \mathrm{y}$ $\left(\neq 0_{\mathrm{A}}\right), \mathrm{t} \in \mathrm{A}, \mathrm{A}$ being the real region $\left(\mathrm{A}, \oplus,,^{*}, \bullet\right)$.

Solution: We have the following equation in the region A:

$$
3 \bullet \mathrm{x} * \mathrm{y}=2 \bullet \mathrm{y} \oplus 3 \bullet \mathrm{t}
$$


Using the properties of region, we then can write

$$
\begin{gathered}
\frac{1}{3} \bullet(3 \bullet \mathrm{x} * \mathrm{y})=\frac{1}{3} \bullet(2 \bullet \mathrm{y} \oplus 3 \bullet \mathrm{t}) \\
\text { or, }\left(\frac{1}{3} \cdot 3\right) \bullet\left(\mathrm{x}^{*} \mathrm{y}\right)=\left(\frac{1}{3} \bullet(2 \bullet y)\right) \oplus\left(\frac{1}{3} \bullet(3 \bullet t)\right) \\
\text { or, } 1_{\mathrm{F}} \bullet\left(\mathrm{x}^{*} \mathrm{y}\right)=\left(\frac{1}{3} \cdot 2\right) \bullet \mathrm{y} \oplus\left(\frac{1}{3} \cdot 3\right) \bullet \mathrm{t} \\
\text { or, } \mathrm{x} * \mathrm{y}=\frac{2}{3} \bullet \mathrm{y} \oplus 1_{\mathrm{F}} \bullet \mathrm{t} \\
\text { or, } \mathrm{x} * \mathrm{y}=\frac{2}{3} \bullet \mathrm{y} \oplus \mathrm{t} \\
\text { or, }(\mathrm{x} * \mathrm{y}) * \mathrm{y}{ }^{-1}=\left(\frac{2}{3} \bullet y \oplus t\right) * \mathrm{y}^{-1} \\
\text { or, } \mathrm{x} *\left(\mathrm{y} * \mathrm{y}^{-1)}=\left(\frac{2}{3} \bullet\left(y * y^{-1}\right)\right) \oplus\left(\mathrm{t} * \mathrm{y}^{-1}\right)\right. \\
\text { or, } \mathrm{x} *\left(\frac{2}{3} \bullet 1_{A} \oplus \frac{t}{y}\right), \mathrm{which} \text { is the solution. } \\
\left(\frac{2}{3} \bullet 1_{A}\right) \oplus\left(\mathrm{t} * \mathrm{y}^{-1}\right)
\end{gathered}
$$

\section{NOTE 3.8 (explanation of the above solution):-}

We analyze now the above solution to the Problem 3.1. Let us imagine a situation that the identity of the algebraic system A (say) in the above Problem 3.1 is unknown to us at this moment, but let us accept that the solution steps are valid in this unknown algebraic system A. In the above solution, we see that:-

There are few steps which are allowed by virtue of the properties of 'vector space' and there are few steps which are allowed by properties of 'division algebra'. It is obvious that a simple 'division algebra' can not give license to all the steps of the above solution (for example, 'compatibility with scalars' is not a licensed step in division algebra, even not the commutative property). Besides that, division operations are executed in the solution and hence A can neither be just an 'algebra over a field' alone nor an 'associative algebra over a field' alone. Consequently, considering the validity of all the involved operations in this solution, this unknown algebra $\mathrm{A}$ has to be at minimum a 'region algebra', not less. Otherwise, the problem can not be solved for $\mathrm{x}$ in $\mathrm{A}$.

\section{Calculus Space and Region Calculus}

It has been justified in the previous section that many of the simple results, formula, equalities, identities, rules etc. of elementary algebra are not valid in general in a group, ring, field, module, linear space, algebra over a field, associative algebra over a field, division algebra, or in any existing algebraic system alone, by their respective definitions and by virtue of their respective properties, but in 'region algebra'. The minimum platform required for practicing elementary algebra is the region algebra. In this section we introduce the notion of 'calculus space' as the minimal structured mathematical space where a new calculus can be developed, and then we introduce the concept of 'multi-dimensional calculus space'. A calculus space is a real region subject to fulfillment of few conditions which are explained here. Without a calculus space, a calculus can not be developed. It is observed that the classical calculus developed independently by Newton and Leibniz is based on the platform of RR region as its own calculus space. The topic is initiated in this section with the prior assumption that in our giant universe or multiverse (if exists), it may happen that the classical calculus of Newton and Leibniz may not be applicable at everywhere in the space. But whatever be the appropriate calculus at some location in this universe (or multiverse), it can only be developed over an appropriate 'calculus space' of its own. The Universe is commonly defined as the totality of existence as far as we people on this earth can think about. The present universe appears to be expanding at an accelerating rate. There are many competing theories about the ultimate fate of the universe. Scientific observation of the universe has led to inferences of its earlier stages too. Physicists remain unsure about what, if anything, preceded the Big Bang. Many refuse to speculate, doubting that any information from any such prior state could ever be accessible. There are various multiverse hypotheses too, in which physicists have suggested that this universe might be one among many universes that likewise exist. If the speculation about the existence of multiverse be accepted to be true, then one question arises: Whether every space of this universe is being governed by the same physical laws and constants throughout most of its extent and history? We consider here the important mathematical system 'Calculus' (developed independently by Newton and Leibniz). John von Neumann said: "The calculus was the first achievement of modern mathematics and it is difficult to overestimate its importance. I think it defines more unequivocally than anything else the inception of modern mathematics, and the system of mathematical analysis, which is its logical development, still constitutes the greatest technical advance in exact thinking". But, can we accept the hypothesis that this classical Calculus is valid in every planet of our solar system or at every space of our universe or at every universe of the multiverse (if exists)? Is our classical Calculus an absolute calculus for everywhere in our universe or multiverse? Does it not get influenced by the facts of relativity. Does it not get influenced at different solar systems or at different spaces of the universe or at different universes of the multiverse where the concept of 'time' and 'distance' are different? In this section we do not (can not) propose any answer to these questions, but we propose the hypothesis that there could be a generalized calculus of which our existing classical calculus is a particular case just. Or, there could be a 
number of new calculus which are siblings to our existing classical calculus. In this quest, we introduce new mathematical notions called by "Calculus Space" and "Region Calculus". A calculus space is a base-platform on which one can develop a new calculus. In other words, a calculus can not be developed without a calculus space, called a base-platform of the calculus. We use the following notations:

$\mathrm{R}=$ set of all real numbers, $\mathrm{R}^{+}=$set of all positive real numbers, $R^{-}=$set of all negative real numbers, $R^{\geq 0}=$ set of all non-negative real numbers. First of all we define two new terms called by 'extended region' and '2-to-1 bijective mapping'.

\section{1. 'Chain Region' and 'Partitioned Region'}

Consider a real region $\mathrm{A}=\left(\mathrm{A}, \oplus,{ }^{*}, \bullet\right)$. Suppose that $\mathrm{A}$ forms a chain with respect to a total order relation (say, denoted by the notation ' $\leq$ '). Then the real region A is called a chain region with respect to the tot al order relation ' $\leq$ '.

A real region $\mathrm{A}=\left(\mathrm{A}, \oplus,{ }^{*}, \bullet\right)$ is called a Partitioned Region if the following conditions are satisfied:

i. A is an infinite region,

ii. A is a chain region with respect to a total order relation ' $\leq$ ', and

iii. the characteristic of $\mathrm{A}$ is zero.

Here $A$ is called a partitioned region because of the fact that it induces a partition $\mathrm{P}_{\mathrm{A}}$ of $\mathrm{A}$ into three mutually disjoint non-null sets $\mathrm{A}^{+}, \mathrm{A}^{-}$and $\left\{0_{\mathrm{A}}\right\}$ such that

i. $\mathrm{A}^{+}=\left\{\mathrm{a}: \mathrm{a} \in \mathrm{A}\right.$ and $\left.0_{\mathrm{A}}<\mathrm{a}\right\}$

ii. $\mathrm{A}^{-}=\left\{\mathrm{a}: \mathrm{a} \in \mathrm{A}\right.$ and $\left.\mathrm{a}<0_{\mathrm{A}}\right\}$.

Clearly, $\forall \mathrm{a} \in \mathrm{A}^{+}, \sim \mathrm{a} \in \mathrm{A}^{-}$and $\forall \mathrm{b} \in \mathrm{A}^{-}, \sim \mathrm{b} \in \mathrm{A}^{+}$.

(Note: It may be recalled from the properties of the chain that: $\mathrm{a}<\mathrm{b}$ iff $\mathrm{a} \leq \mathrm{b}$ and $\mathrm{a} \neq \mathrm{b}$, where " $\leq$ " is the total order relation of the chain $A$, and similarly $a>b$ iff $b \leq a$ and $b \neq a$ ).

This partition $\mathrm{P}_{\mathrm{A}}$, once made, is regarded as an absolute partition of the region A corresponding to its total order relation ' $\leq$ 'in the sense that this partition generates the sign of every object of the complete region A, positive or negative, which will remain absolute for the complete literature of the corresponding region mathematics.

\subsection{Extended Region}

Consider a partitioned region $\mathrm{A}=\left(\mathrm{A}, \oplus,,^{*}, \bullet\right)$. If we now include two more objects $+\propto_{\mathrm{A}}$ and $-\propto_{\mathrm{A}}$ in $\mathrm{A}$ as two guests, then the set $A \cup\left\{+\propto_{A},-\propto_{A}\right\}$ is called to be an extended region.

Here $+\propto_{\mathrm{A}}=\frac{x_{A}}{0_{A}}$ where $x_{A}\left(\neq 0_{\mathrm{A}}\right)$ is any positive object, and $-\propto_{\mathrm{A}}=\frac{z_{A}}{0_{A}}$ where $z_{A}\left(\neq 0_{\mathrm{A}}\right)$ is any negative object. Note that an extended region is not a region. But whenever we say that $\mathrm{A}$ is an extended region, it will imply that $\mathrm{A}$ is a region and two infinities are also included to it as permanent guests.

\subsection{2-to-1 Bijective Mapping}

Consider two non-null sets $\mathrm{X}$ and $\mathrm{Y}$. A function $\mathrm{f}: \mathrm{X} \rightarrow \mathrm{Y}$ is said to be a '2-to-1 Bijective Mapping' if (i) $\mathrm{f}$ is onto, and

(ii) $\forall \mathrm{y} \in \mathrm{Y}, \exists$ two and only two distinct (not same) elements $\mathrm{x}_{1}$ and $\mathrm{x}_{2}$ in $X$ such that $\mathrm{f}\left(\mathrm{x}_{1}\right)=\mathrm{y}=\mathrm{f}\left(\mathrm{x}_{2}\right)$.

For example, the function $\mathrm{f}: \mathrm{R}-\{0\} \rightarrow \mathrm{R}^{+}$given by $\mathrm{f}(\mathrm{x})=$ $\mathrm{x}^{2}$ is a 2-to-1 Bijective Mapping.

\subsection{Calculus Space}

Consider a partitioned region $\mathrm{A}=\left(\mathrm{A}, \oplus,{ }^{*}, \bullet\right)$. Then $\mathrm{A}$ forms a Calculus Space if the following conditions are satisfied:

(i) A is an extended real region.

(ii) $\mathrm{A}$ is a normed complete metric space with respect to a norm $\|$.$\| and the corresponding induced metric \rho(\mathrm{x}, \mathrm{y})=$ $\|\mathrm{x} \sim \mathrm{y}\|$, (i.e. $\|\mathrm{x}\|=\rho\left(\mathrm{x}, 0_{\mathrm{A}}\right)$ ).

(iii) The norm $\|\cdot\|$ is a 2-to-1 bijective mapping from $\mathrm{A}-$ $\left\{0_{\mathrm{A}}\right\}$ to $\mathrm{R}^{+}$.

\subsubsection{What Is the 'Calculus Space' for Newton and Leibniz Calculus}

As a particular instance, if we choose the region A to be the RR region (see Example 3.4 presented earlier) considering it as a partitioned region with respect to the crisp order relation "Less Than or Equal To" denoted by the notation " $\leq$ ", and if we choose $\|\mathrm{x}\|=|\mathrm{x}|$ in RR, where $\rho(\mathrm{x}$, $y)=\|x-y\|=|x-y|$, then the corresponding region calculus happens to be the classical calculus (developed independently by Newton and Leibniz).

\subsubsection{A simple Division Algebra Can not Form a Calculus Space in General}

The set $\mathrm{R}$ of real numbers is so interesting that it very comfortably forms the region $\mathrm{RR}$; and the region $\mathrm{RR}$ is so beautiful that it satisfies all the necessary conditions to form a Calculus Space (an eligible platform on which a calculus can be developed). This is just a coincidence for the case of division algebra $\mathrm{R}$, because a simple division algebra by definition does not have so much capability. Consequently, it is clear now that the classical calculus developed independently by Newton and Leibniz happens to be on the particular calculus space RR with respect to a particular order relation "Less Than or Equal To" denoted popularly by the notation " $\leq$ ".

The following facts may be recalled [25, 27] that the metric $\rho$ associated with the norm $\|\cdot\|$ i.e. the metric $\rho$ (x, $\mathrm{y})=\|\mathrm{x} \sim \mathrm{y}\|$ has the following special properties:

(i) 'Translation Invariance': i.e. $\forall \mathrm{z} \in \mathrm{A}$ we have $\rho(\mathrm{x} \oplus \mathrm{z}$, $\mathrm{y} \oplus \mathrm{z})=\rho(\mathrm{x}, \mathrm{y})=\|\mathrm{x} \sim \mathrm{y}\|$, and

(ii) 'Homogeniety': i.e. $\forall \mathrm{r} \in \mathrm{R}$ we have $\rho(\mathrm{r} \bullet \mathrm{x}, \mathrm{r} \bullet \mathrm{y})=$ $|\mathrm{r}| \cdot\|\mathrm{x} \sim \mathrm{y}\|=|\mathrm{r}| \cdot \rho(\mathrm{x}, \mathrm{y})$.

Although these two beautiful properties were established much later than the discovery of the classical calculus, but these were fortunately true in the 'Calculus Space' of Newton and Leibniz calculus, by default.

\subsection{Complete Region}

A real region which forms a calculus space is called a "complete region". 
We will introduce later that by a complete region, we will always mean one-dimensional complete region (1-D complete region). For instance, the region RR is a complete region with respect to the crisp order relation "Less Than or Equal To" denoted by the notation " $\leq$ " and the metric $\rho$ (x, $y)=\|x \sim y\|=|x-y|$, where the norm is the classical norm defined over $\mathrm{R}$.

The collection of all the complete regions is called the complete region universe $\Sigma$.

\subsection{Positive Object, Negative Object and Object Linear Continuum Line}

Consider a complete region $\mathrm{A}=(\mathrm{A}, \oplus, *, \bullet)$. The elements of $\mathrm{A}^{+}$are said to be positive objects and the elements of $\mathrm{A}^{-}$are said to be negative objects. The object $0_{\mathrm{A}}$ is neither in $\mathrm{A}^{+}$nor in $\mathrm{A}^{-}$, and so we say that $0_{\mathrm{A}}$ is neither a positive object nor a negative object. The attribute of being positive or negative is called the sign of the object, and $0_{\mathrm{A}}$ is not considered to have a sign of its own. For a given calculus space $\mathrm{A}$, a line $\mathrm{XX}^{1}$ can be drawn with all positive objects lying upon it to the right of $0_{\mathrm{A}}$, and all negative objects lying upon it to the left of $0_{\mathrm{A}}$ as shown in Figure 1. Thus the 'positive direction' of $\mathrm{X}$ axis and the 'negative direction' of $\mathrm{X}$-axis can be well understood and the line which the objects of the complete region $\mathrm{A}$ is considered to lie upon is called the Object Linear Continuum Line (see Figure 1 below).

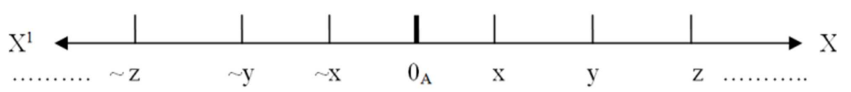

Fig. 1. Objects linear continuum line of the complete region A, a general view.

Thus, any point on the Object Linear Continuum Line of the complete region $\mathrm{A}$ is called an object point of $\mathrm{A}$.

We use the following notations in our work here:

$\mathrm{A}^{+}=$set of all positive objects of the complete region $\mathrm{A}$,

$\mathrm{A}^{-}=$set of all negative objects of the complete region $\mathrm{A}$,

$\mathrm{A}^{\geq 0}=$ set of all non-negative objects of the complete region $\mathrm{A}$.

For developing a new calculus, be it in a two dimensional coordinate system, or in an n-dimensional coordinate system, at least one calculus space is required. Consider the object linear continuum line and the corresponding X-axis. Since the region $\mathrm{A}$ is complete, there are no "points missing" from it (inside or at the boundary). Since A is a chain, every object of $\mathrm{A}$ has a unique address on this object linear continuum line and conversely i.e. corresponding to every address (point) on this object linear continuum line there is a unique object of the region $\mathrm{A}$.

Consider a point $\mathrm{x}$ on the $\mathrm{X}$-axis of the object linear continuum line corresponding to the calculus space $\mathrm{A}$. Then for an infinitesimal small positive object $\Delta \mathrm{x}$, the point $(\mathrm{x} \oplus$ $\Delta \mathrm{x})$ will be at a distance $\|\Delta \mathrm{x}\|$ from the point $\mathrm{x}$ along the positive direction of $\mathrm{X}$-axis and the point $(\mathrm{x} \sim \Delta \mathrm{x})$ will be at a distance $\|\Delta \mathrm{x}\|$ from the point $\mathrm{x}$ along the negative direction of $\mathrm{X}$-axis. By distance between two objects $\mathrm{x}$ and $\mathrm{y}$ lying upon the $\mathrm{XX}^{1}$ Object Linear Continuum Line of the complete region $\mathrm{A}$, we mean the corresponding metric distance $\rho(\mathrm{x}, \mathrm{y})$ of the normed complete metric space A.

For example, see a collection of consecutive equi-spaced points on the object line as shown in the Figure 2 below.

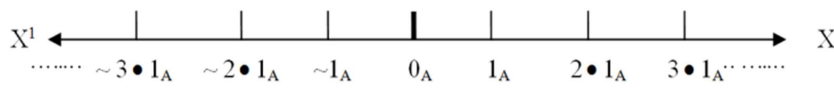

Fig. 2. Object Linear Continuum Line of the complete region $A$ with few consecutive equi-spaced object points.

The term 'equi-spaced' in the caption of Figure 2 is well understood in the sense of the corresponding metric (or norm) of the complete region A, i.e. for any real integer $\mathrm{r}, \rho$

$\left(\mathrm{r} \bullet 1_{\mathrm{A}},(\mathrm{r}+1) \bullet 1_{\mathrm{A}}\right)=$ constant (independent of $\mathrm{r}$ ), in the complete region A.

Example 4.1

If we choose the region $A$ to be the RR region (see Example 3.4 presented earlier) which is a partitioned region with respect to the crisp order relation "Less Than or Equal To" denoted by the notation " $\leq$ ", and if we choose $\|x\|=|x|$ in RR, where $\rho(\mathrm{x}, \mathrm{y})=\|\mathrm{x}-\mathrm{y}\|=|\mathrm{x}-\mathrm{y}|$, then the corresponding $\mathrm{X}$-axis is the classical $\mathrm{X}$-axis popularly used by us in the Cartesian coordinate system, the corresponding linear object continuum is the classical real continuum and the corresponding region calculus happens to be the classical calculus (developed independently by Newton and Leibniz).

It will be mistake if we say that the classical calculus is based on the field $\mathrm{R}$ of real numbers (of course, considering the extended real-axis). Actually it is neither the field $\mathrm{R}$ nor the division algebra $\mathrm{R}$, but it is the region $\mathrm{R}$ (which is called by $R R$ region in the region algebra). Interestingly, the division algebra $\mathrm{R}$ satisfies few additional properties trivially (not by virtue of the definition and properties of 'division algebra' as mentioned in Section 2, an important issue which is to be seriously noted). And by fulfilling these additional properties, $\mathrm{R}$ well qualifies to become a real region too. Consequently the classical calculus never faced any computational constraints or invalidity even assuming $\mathrm{R}$ to be a field or division algebra just. Fortunately it is a coincidence that $\mathrm{R}$ does also form a complete region! Otherwise the classical calculus would not have reached the extremely rich today's level, rather it would have become blocked somewhere at some time much earlier in its so long beautiful network of journey.

\subsection{Developing a New Calculus: Region Calculus}

We are aware about the history, development and growth of the classical calculus since its inception (happened to be developed on the calculus space RR). We define that a calculus developed on a calculus space is called a Region Calculus.

Let us consider a calculus space A. Suppose that we want to develop now a new region calculus in the calculus space A. For this purpose, the basic concepts of any new calculus (of a new differential calculus) are: limit, continuity, differentiability of a function of objects, etc. which we need to introduce first of all in the calculus space A analogous to 
the classical way of Newton and Leibniz style.

\subsubsection{What do You Mean by " $x \rightarrow a$ "}

Consider an object variable $\mathrm{x}$ over the calculus space $\mathrm{A}=$ $\left(\mathrm{A}, \oplus,{ }^{*}, \bullet\right)$. Let $\mathrm{a} \in \mathrm{A}$ be a fixed object. Suppose that $\mathrm{x}$ assumes successive values, some of them for example are: (a $\left.\oplus 0.1 \bullet 1_{\mathrm{A}}\right),\left(\mathrm{a} \oplus 0.01 \bullet 1_{\mathrm{A}}\right),\left(\mathrm{a} \oplus 0.001 \bullet 1_{\mathrm{A}}\right),(\mathrm{a} \oplus 0.0001 \bullet$ $\left.1_{\mathrm{A}}\right), \ldots \ldots$ in its course to get close and close to the object a.

Obviously, as x passes through these values, the value $\rho$

$(\mathrm{x}, \mathrm{a})$ becomes less and less and become so small that for any positive real number $\varepsilon$, no matter however small, $\rho$ (x, a) $<$ $\varepsilon$ is satisfied. Let us express this situation using the notation "x $\rightarrow$ a+" which means that the object variable $x$ approaches the fixed object a from the right hand side of a (see Figure 3).

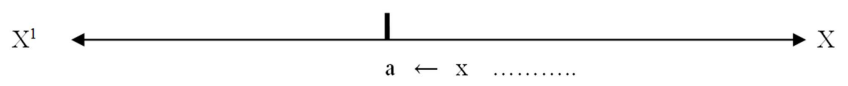

Fig. 3. On the Object Linear Continuum Line: $x \rightarrow a+$

Similarly, suppose that $\mathrm{x}$ assumes successive values, some of them for example are:

$$
\begin{gathered}
\left(\mathrm{a} \sim 0.1 \bullet 1_{\mathrm{A}}\right),\left(\mathrm{a} \sim 0.01 \bullet 1_{\mathrm{A}}\right),\left(\mathrm{a} \sim 0.001 \bullet 1_{\mathrm{A}}\right), \\
\left(\mathrm{a} \sim 0.0001 \bullet 1_{\mathrm{A}}\right), \ldots \ldots .
\end{gathered}
$$

in its course to get close and close to the object a.

Obviously, as $\mathrm{x}$ passes through these successive values, the value $\rho(\mathrm{x}, \mathrm{a})$ becomes less and less and become so small that for any positive real number $\varepsilon$, no matter however small, $\rho(\mathrm{x}, \mathrm{a})<\mathcal{E}$ is satisfied. Let us express this situation using the notation " $\mathrm{x} \rightarrow \mathrm{a}-$ " which means that the object variable $\mathrm{x}$ approaches the fixed object a from the left hand side of a(see Figure 4).

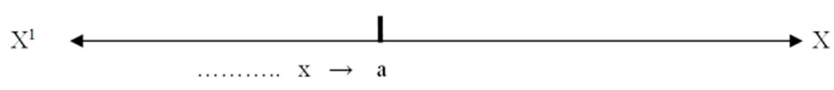

Fig. 4. Object Linear Continuum Line: $x \rightarrow a$-.

By the expression " $x$ tends to a" symbolically written as " $\mathrm{x}$ $\rightarrow$ a", we mean that given any real $\varepsilon>0$ no matter however small, the successive values of $\mathrm{x}$ ultimately satisfy the inequality $0<\rho(\mathrm{x}, \mathrm{a})<\mathcal{E}$. It is to be noted that if " $\mathrm{x} \rightarrow \mathrm{a}$ " then $\rho(\mathrm{x}, \mathrm{a}) \neq 0$, i.e. $\mathrm{x} \neq \mathrm{a}$.

\subsubsection{Neighborhood of an Object Point on the Object Linear Continuum Line}

Consider an object a on the Object Linear Continuum Line of the calculus space $\mathrm{A}=\left(\mathrm{A}, \oplus,{ }^{*}, \bullet\right)$. Let $\delta>0$ be a real number. Then the $\delta$-neighborhood of the object a is defined by the set $\mathrm{N}_{\delta}(\mathrm{a})$ of objects given by $\mathrm{N}_{\delta}(\mathrm{a})=\{\mathrm{x}: \mathrm{x} \in \mathrm{A}$ and $\rho(\mathrm{x}, \mathrm{a})<\delta\}$.

Here $\mathrm{N}_{\delta}(\mathrm{a}) \subseteq \mathrm{A}$, and obviously $\mathrm{N}_{\delta}(\mathrm{a}) \neq \varphi$.

\subsubsection{Limit of a Function}

Let $\mathrm{X}$ and $\mathrm{Y}$ be two non-null subsets of the calculus space $\mathrm{A}=\left(\mathrm{A}, \oplus,{ }^{*}, \bullet\right)$ and let $\mathrm{f}$ be a function $\mathrm{f}: \mathrm{X} \rightarrow \mathrm{Y}$ which is actually an object valued function of object variable. Then $\mathrm{f}(\mathrm{x})$ is said to have a limit 1 in $\mathrm{Y}$ if for any pre-assigned real number $\varepsilon>0$, no matter however small, $\exists$ a real number $\delta>$
0 such that $\rho(\mathrm{f}(\mathrm{x}), 1)<\varepsilon$ whenever $0<\rho(\mathrm{x}, \mathrm{a})<\delta$.

We write symbolically as: $\lim _{x \rightarrow a} f(x)=1$, i.e. $\mathrm{f}(\mathrm{x}) \rightarrow 1$ as $\mathrm{x}$ $\rightarrow$ a.

Problem 4.1

Show that $\lim _{x \rightarrow 2 \bullet 1_{A}} 5 \bullet x=10 \bullet 1_{\mathrm{A}}$ in the calculus space $\mathrm{A}=$ $(\mathrm{A}, \oplus, *, \bullet)$.

Solution: Given real $\varepsilon>0$, no matter however small, we need to find out real $\delta>0$ such that

$\rho\left(5 \bullet \mathrm{x}, 10 \bullet 1_{\mathrm{A}}\right)<\mathcal{E}$ whenever $0<\rho\left(\mathrm{x}, 2 \bullet 1_{\mathrm{A}}\right)<\delta$.

i.e. $\left\|5 \bullet \mathrm{x} \sim 10 \bullet 1_{\mathrm{A}}\right\|<\varepsilon$ whenever $0<\left\|\mathrm{x} \sim 2 \bullet 1_{\mathrm{A}}\right\|<\delta$.

i.e. 5. $\left\|\mathrm{x} \sim 2 \bullet 1_{\mathrm{A}}\right\|<\varepsilon$ whenever $0<\left\|\mathrm{x} \sim 2 \bullet 1_{\mathrm{A}}\right\|<\delta$, using properties mentioned in subsection-4.4.1.

Now if we choose $\delta=\varepsilon / 5$, our definition is satisfied.

Hence $\lim _{x \rightarrow 2 \bullet 1_{A}} 5 \bullet x=10 \bullet 1_{\mathrm{A}}$ in the calculus space A.

Problem 4.2

Show that $\lim _{x \rightarrow 3 \bullet 1_{A}} \frac{x^{2} \sim 9 \bullet 1_{A}}{x \sim 3 \bullet 1_{A}}=6 \bullet 1_{\mathrm{A}}$ in the calculus space $\mathrm{A}=(\mathrm{A}, \oplus, *, \bullet)$.

Solution: Given $\varepsilon>0$, no matter however small, we need to find out $\delta>0$ such that

$$
\rho\left(\frac{x^{2} \sim 9 \bullet 1_{A}}{x \sim 3 \bullet 1_{A}}, 6 \bullet 1_{A}\right)<\varepsilon \text { whenever } 0<\rho\left(\mathrm{x}, 3 \bullet 1_{\mathrm{A}}\right)<\delta .
$$

i.e. $\left\|\frac{x^{2} \sim 9 \bullet 1_{A}}{x \sim 3 \bullet 1_{A}} \sim 6 \bullet 1_{A}\right\|<\varepsilon$ whenever $0<\left\|\mathrm{x} \sim 3 \bullet 1_{\mathrm{A}}\right\|<\delta$.

Since $\mathrm{x} \rightarrow 3 \bullet 1_{\mathrm{A}}$ therefore $\mathrm{x} \neq 3 \bullet 1_{\mathrm{A}}$ and hence $\left(\mathrm{x} \sim 3 \bullet 1_{\mathrm{A}}\right)$ $\neq 0_{\mathrm{A}}$.

Therefore, Cancellation Laws of region algebra can be applied to get the following result:

$\left\|\left(\mathrm{x} \oplus 3 \bullet 1_{\mathrm{A}}\right) \sim 6 \bullet 1_{\mathrm{A}}\right\|<\mathcal{E}$ whenever $0<\left\|\mathrm{x} \sim 3 \bullet 1_{\mathrm{A}}\right\|<\delta$.

i.e. $\left\|\mathrm{x} \sim 3 \bullet 1_{\mathrm{A}}\right\|<\mathcal{E}$ whenever $0<\left\|\mathrm{x} \sim 3 \bullet 1_{\mathrm{A}}\right\|<\delta$.

Now if we choose $\delta=\varepsilon$, our definition is satisfied. Hence the result.

\subsection{Multi-dimensional Calculus Space and Multi-dimensional Region Calculus}

The calculus space discussed so far is basically one dimensional calculus space (1-D calculus space) and the corresponding region calculus is also one dimensional. It is because of the reason that in a calculus space any variable $\mathrm{x}$ can vary/move along a straight line only. By a 'complete region' we shall mean that it is corresponding to 1-D calculus space. By the simple terms: calculus space, region calculus, complete region, we shall always mean here the same in onedimensional.

In this section we introduce the concept of 'Multidimensional Calculus Space' as a generalization of the concept of 'calculus space'. In a two-dimensional calculus 
space (2-D calculus space), a variable $\mathrm{z}$ can move along a curve on a plane. The corresponding region calculus is called a 2-D region calculus. In a three-dimensional calculus space (3-D calculus space), a variable w can move along a curve on a 3-D space. The corresponding region calculus is called a 3$\mathrm{D}$ region calculus. Similarly, in an $\mathrm{n}-\mathrm{D}$ calculus space, a variable $\mu$ can move along a curve on a n-D hyperspace. The corresponding region calculus is called an $\mathrm{n}-\mathrm{D}$ region calculus. Instead of extended real region there could be more number of infinities leading to 'multi-extended real region'. The notion of multi-extended real region needs to be studied in depth in future. However, let us call a partitioned region to be a multi-extended region if it has more than two infinities. We now define an n-to-1 Bijective Mapping.

\subsection{1. n-to-1 Bijective Mapping}

Consider two non-null sets $\mathrm{X}$ and $\mathrm{Y}$. A function $\mathrm{f}: \mathrm{X} \rightarrow \mathrm{Y}$ is said to be a ' $n$-to-1 Bijective Mapping' if

(i) $\mathrm{f}$ is onto, and

(ii) $\forall \mathrm{y} \in \mathrm{Y}, \exists \mathrm{a}$ unique subset $\mathrm{S}_{\mathrm{y}}$ of $\mathrm{X}$ of cardinality $\mathrm{n}$ ( $>$ 2) such that $\forall \mathrm{x} \in \mathrm{S}_{\mathrm{y}}$ we have $\mathrm{f}(\mathrm{x})=\mathrm{y}$.

Here, $n$ could be finite positive integer $(>2)$ or infinity.

For example, the function $\mathrm{f}: \mathrm{C}-\{0\} \rightarrow \mathrm{R}^{+}$given by $\mathrm{f}(\mathrm{z})=$ $|z|^{2}$ is a n-to-1 Bijective Mapping.

\subsubsection{Multi-dimensional Calculus Space}

Consider a partitioned region $\mathrm{A}=(\mathrm{A}, \oplus, *, \bullet)$. Then $\mathrm{A}$ forms a Multi-dimensional Calculus Space if the following conditions are satisfied:

(i) A is a multi-extended real region.

(ii) $\mathrm{A}$ is a normed complete metric space with respect to a norm $\|$.$\| and the corresponding induced$

metric $\rho(\mathrm{x}, \mathrm{y})=\|\mathrm{x} \sim \mathrm{y}\|$, (i.e. $\|\mathrm{x}\|=\rho\left(\mathrm{x}, 0_{\mathrm{A}}\right)$ ).

(iii) The norm $\|\cdot\|$ is a n-to- 1 bijective mapping from $\mathrm{A}-$ $\left\{0_{\mathrm{A}}\right\}$ to $\mathrm{R}^{+}$for some fixed integer $\mathrm{rn}>2$.

\subsection{3. $n-D$ Complete Region}

A real region which forms a n-D calculus space is called a "n-D complete region".

A calculus developed out of n-dimensional calculus space is called by $\mathrm{n}$-dimensional region calculus. It may happen that a region can not form an $\mathrm{n}_{1}$-dimensional calculus space, but can well form an $n_{2}$-dimensional calculus space. In other words, a region may not form an $\mathrm{n}_{1}$-dimensional region calculus, but may well form an $\mathrm{n}_{2}$-dimensional region calculus.

It is to be carefully noted that as per definition (see Section 2), a Division Algebra is not a region in general. Consequently a Division Algebra can not become a Calculus Space in general even if it satisfies all the conditions of Calculus Space. Given any region $\mathrm{G}=\left(\mathrm{G}, \oplus,{ }^{*}, \bullet\right)$ over the field $(\mathrm{R},+,$.$) , one can immediately attempt to explore$ whether $\mathrm{G}$ forms a calculus space with respect to some norm $\|$.$\| and a total order relation' \leq$ '. If $\mathrm{G}$ forms a calculus space, then a new calculus can be developed in $\mathrm{G}$. The set $\mathrm{C}$ of complex numbers does not satisfy the required conditions to become a calculus space with respect to its popular norm $|z|=\sqrt{z \bar{z}}=\sqrt{x^{2}+y^{2}}$. Consequently, no 1-D region calculus can be developed in the region $\mathrm{C}$.

However, in our future research work we need to explore whether $\mathrm{C}$ forms a multi-dimensional calculus space (say, 2$\mathrm{D}$ calculus space) with respect to its popular norm $|z|=\sqrt{z \bar{z}}$ so that a 2-D region calculus can be developed in C. The set of triangular fuzzy numbers (trapezoidal fuzzy numbers) does not form a region $[8,9]$ with respect to its existing known operators and consequently it can not offer any region calculus of any dimension to us.

\subsection{An Interesting Question Arises: How Many Distinct 1-D Complete Regions Exist Mathematically in Region Mathematics}

To answer this question, first of all we see that given a region $\mathrm{A}=\left(\mathrm{A}, \oplus,{ }^{*}, \bullet\right)$ over the field $(\mathrm{F},+,$.$) there could be$ more regions corresponding to the same set A over the same set $\mathrm{F}$ but with different operators $\oplus, *$, - and + , respectively.

Even if $\mathrm{P}=\left(\mathrm{A}, \oplus,{ }^{*}, \bullet\right)$ be a given fixed complete region with respect to the total order relation ' $\leq$ ' and the norm $\|$.$\| ,$ there could be another distinct complete region $\mathrm{Q}=(\mathrm{A}, \oplus, *$, -) with respect to a different total order relation or with respect to a different norm or with respect to different pair of total order relation and norm both. There could be many more such complete region $\left(\mathrm{A}, \oplus,,^{*}, \bullet\right)$ in similar ways. However, we will explore this in depth in our future research work.

Thus a given region $\mathrm{A}=\left(\mathrm{A}, \oplus,{ }^{*}, \bullet\right)$ over the field $(\mathrm{F},+,$. may produce more than one distinct complete regions (even retaining the set $A$, retaining the set $F$ and retaining the operators $\oplus, *, \bullet$ and + ,. unchanged), but with different total order relations and different norms, subject to fulfillment of the definition of one dimensional region calculus.

For example, consider the Newton Calculus which is based upon the complete region RR but with respect to the crisp order relation "Less Than or Equal To" denoted by the notation " $\leq$ " and the classical norm $\|$.$\| defined by \|x\|=|x|$ in $\mathrm{RR}$, where the corresponding metric is given by $\rho(\mathrm{x}, \mathrm{y})$ $=\|x-y\|=|x-y|$.

Now, for any real number $\mathrm{k}>0$ we can define a new norm $\|\cdot\|_{\text {new }}$ over the region RR as below:

$$
\|x\|_{\text {new }}=\mathrm{k}|\mathrm{x}|
$$

It can be observed that the region RR in this case forms a new one dimensional calculus space with respect to this new norm $\|\cdot\|_{\text {new }}$ and the corresponding metric $\rho_{\text {new }}$ given by $\rho_{\text {new }}(x, y)=\|x \sim y\|_{\text {new }}=\mathrm{k}|\mathrm{x}-\mathrm{y}|$, even retaining the same crisp order relation "Less Than or Equal To" $(\leq)$. Thus we can define infinite number of distinct norms mathematically and infinite number of distinct corresponding metrics. Thus, even retaining the same total order relation we can define infinite number of distinct 1-D region calculus mathematically in real situation, besides the classical 1-D 
region calculus 'Newton Calculus' based upon the real region $\mathrm{RR}$, but with respect to the crisp order relation "Less Than or Equal To" denoted by the notation " $\leq$ " and the classical norm $\|$.$\| defined by \|x\|=|x|$ in $R R$, where the corresponding metric is given by $\rho(\mathrm{x}, \mathrm{y})=\|\mathrm{x}-\mathrm{y}\|=|\mathrm{x}-\mathrm{y}|$. Thus we can define infinite number of distinct 1-D complete regions mathematically.

\section{Conclusion}

The work on "Region Mathematics" was not initiated in my mind with any pre-posed problem or plan. I did not have any pre-proposed synopsis for it. It was an accidental development in my mind while I observed that in general the existing standard algebraic systems alone viz. groups, rings, modules, fields, linear spaces, algebra over a field, associative algebra over a field, division algebra, etc. can not validate many of the fundamental and classical equalities, identities, expressions, equations, formulas, results of "elementary algebra" (of secondary school level or higher level) by virtue of their respective definitions and properties. Four examples are presented and explained in Case- 4 in section-3.1, but there are infinite number of examples. The 'Algebra' as a subject needs to identify an appropriate algebraic system of it on the platform of which the most practiced classical equalities, identities, expressions, equations, formulas, results of elementary algebra stand valid, can be computed and verified to be true. Yes, it is fact that an infinite number of algebraic systems can be defined by the algebraists, but the objective of this work is not to introduce a new one so. The attempt made in this work is a genuine requirement for the subject Mathematics, and in fact a very important and truly mandatory requirement. By 'Mathematics' we mean a vast family consisting of a large number of giant members viz. Algebra, Number Theory, Arithmetic, Geometry, Trigonometry, Calculus, Mechanics, Astronomy,... etc. to list a few only out of many. The existing dragon volume of literatures on Mathematics developed so far is just a particular case of "Region Mathematics". Consequently, it will not be appropriate if we say that the "Region Mathematics" is simply an extension of the existing Mathematics. Because the "Region Mathematics" is an integrated figure of many such Mathematics (of which the existing Mathematics is just one member). The sole objective of this work is to introduce "Region Mathematics" which is initiated by unearthing a new algebraic system which provides the minimal platform (unlike any other existing classical algebraic system alone, in general) to make the fundamental and classical equalities, identities, expressions, equations, formulas, results, etc. valid (i.e. can be computed and verified); to provide the practitioner of algebra an algebraic right to use the standard and most practiced equalities, identities, expressions, equations, formulas, results, etc of it fluently in the everyday algebraic computations. Identifying this algebraic system and then defining it uniquely with an independent self-identity is therefore important for us. Consequently in Section-3 a new but very sound and complete algebraic system called by "region" has been introduced. Various properties of the algebraic system 'regions' are studied, and a lot of characterizations is done. Region is the most practiced algebra in the study of Science, Technology, Engineering, etc. The scientists, engineers, mathematicians work fluently always being based upon a platform of an hidden algebraic system which is at least a region. Region can be visualized using permutation/combination of various existing classical brands of algebraic systems. Considering the enormous unique potential of "Region Algebra" to give license to the mathematicians to practice the existing simple and useful results, equalities, identities, formulas etc. of elementary algebra, we can not ignore the deserving and genuine claim of "Region Algebra" to have a self independent identity.

Philosophically, if we consider the evolution of various algebraic systems, in particular considering their flexible roles and volume of contributing capabilities towards the subjects from 'elementary algebra' to 'higher algebra', we could visualize the unique location of "Region" as mentioned below, which has been unearthed in this work:-

$$
\begin{gathered}
\text { Group } \rightarrow \text { Ring } \rightarrow \text { Field } \rightarrow \text { Linear Space } \rightarrow \text { Division } \\
\text { Algebra } \rightarrow \text { Region. }
\end{gathered}
$$

In Section-4 we then introduce the branch of 'Region Calculus' in Region Mathematics by defining 'Calculus Space'. The classical calculus developed independently by Newton and Leibniz is based on the set $\mathrm{R}$ of real numbers, extended with two infinities, and then took its shape further with functions of complex variables, vector calculus, tensor calculus, etc. The growth of classical calculus at every stage required fluent applications of various properties of the set $\mathrm{R}$ of real numbers. Using the properties of a 'field' or a 'division algebra' or any existing algebra other than region algebra, the classical calculus can not have the validity of its all fluent results. Fortunately the set $\mathrm{R}$ is a trivial example of real region and the mathematicians enriched the classical calculus using the properties of region $\mathrm{R}$, although 'unknowingly'. It is fact because of the reason that the development of the classical calculus can not be agreed by virtue of the definition and properties of any existing brand of standard algebraic system of Algebra, but by virtue of the definition and properties of regions at minimum.

One of the major breakthrough in Region Mathematics is that we have precisely identified: 'What are the minimum properties which need to be satisfied by a set A so that a calculus can be developed over A?'. Consequently we have introduced the notion of 'calculus space' as a general minimal platform on which a calculus can be developed. It has been explained how the platform $\mathrm{R}$ of classical calculus forms a calculus space. For a non-example, the set of all triangular fuzzy numbers do not form a real region with respect to its commonly used operators, and hence can not open any platform to develop any fuzzy differential calculus and fuzzy integral calculus over it in the style of the classical calculus. The requirements are precisely identified as a checklist before making any attempt to develop any new 
calculus over a given set. This work of Regional Calculus is initiated with a prior intuitionistic assumption that the classical calculus may not be applicable successfully at everywhere of our universe system or of the multiverse system (if exists). We presume here that our future computations (be it in this solar system or in other, be it in this universe or in other of the multiverse) may not be sufficiently covered by or compatible with our classical calculus. Consequently, the very first job is to define the general structure of a mathematical space which is a minimum requirement for making an attempt to develop any new calculus over it. It is justified that mathematically there are infinite number of distinct complete regions exist in Region Mathematics, there are infinite number of distinct 1$\mathrm{D}$ region calculus exist in Region Mathematics. Then we generalize the concept of calculus space by defining 'multidimensional calculus space'. The simple term calculus space is basically one dimensional calculus space (1-D calculus space) and the corresponding region calculus is also one dimensional region calculus. In a calculus space any variable $x$ can vary/move along a straight line only, i.e. if $x \rightarrow a$ in a complete region, it means that $\mathrm{x}$ is being driven along a straight line. The concept of 'Multi-dimensional Calculus Space' is a generalization of the concept of 'calculus space'. In a two-dimensional calculus space (2-D calculus space), a variable $\mathrm{z}$ can move along a curve on a plane. The corresponding region calculus is called a 2-D region calculus. In a three-dimensional calculus space (3-D calculus space), a variable $w$ can move along a curve on a 3 -D space. The corresponding region calculus is called a 3-D region calculus. Similarly, in an $n-D$ calculus space, a variable $\mu$ can move along a curve on a $n-D$ hyperspace. The corresponding region calculus is called an n-D region calculus (i.e. n-dimensional region calculus). It may happen that a region can not form an $\mathrm{n}_{1}$-dimensional calculus space, but can well form an $\mathrm{n}_{2}$ dimensional calculus space. In other words, a region may not form an $n_{1}$-dimensional region calculus, but may form an $n_{2}$ dimensional region calculus. It is to be carefully noted that mathematically an arbitrary Division Algebra (see Section-2) is not a region in general by virtue of its definition and properties. Consequently an arbitrary Division Algebra can not qualify to become a Calculus Space in general. The proposed theory of Region Calculus helps us to study for any arbitrary region $\mathrm{G}=(\mathrm{G}, \oplus, *, \bullet)$ over the field $(\mathrm{R},+,$.$) to$ explore whether $G$ forms a calculus space with respect to a suitable norm $\|$.$\| and a suitable total order relation ' \leq$ '. If $\mathrm{G}$ forms a calculus space, then a new calculus can be developed in $\mathrm{G}$. However, the set $\mathrm{C}$ of complex numbers does not satisfy the required conditions to become a calculus space with respect to its popular norm $|z|=\sqrt{z \bar{z}}=\sqrt{x^{2}+y^{2}}$. Consequently, no 1-D region calculus can be developed in the region C. However, in our future research work we need to explore whether $\mathrm{C}$ forms a multi-dimensional calculus space (2-D calculus space) with respect to its popular norm $|z|=\sqrt{z \bar{z}}$ so that a 2-D region calculus can be developed in $\mathrm{C}$. The set of triangular fuzzy numbers (trapezoidal fuzzy numbers) does not form a region $[8,9]$ with respect to its existing known operations, and consequently it can not offer any region calculus of any dimension to us. In the sequel of this present work done in Part-2 [7] on the new direction in mathematics called by "Region Mathematics", we introduce "Theory of Objects", a new kind of "Theory of Numbers" reshaping the existing Elementary Number Theory, and "Region Geometry" (which generates the classical geometry as one of its particular instance).

\section{References}

[1] Althoen, S. C. and Kugler, L. D.: When Is $\mathrm{R}^{2}$ a Division Algebra?. American Math. Monthly. Vol. 90. 625-635 (1983).

[2] Artin, Michael.: Algebra. Prentice Hall, New York. (1991)

[3] Biswas, Ranjit.: Region Algebra, Theory of Objects \& Theory of Numbers. International Journal of Algebra. Vol. 6(8). 1371-1417 (2012).

[4] Biswas, Ranjit.: Calculus Space. International Journal of Algebra. Vol. 7(16). 791-801 (2013).

[5] Biswas, Ranjit.: Region Algebra. Information. Vol. 15(8). 3195-3228 (2012).

[6] Biswas, Ranjit.: "Theory of Numbers" of a Complete Region. Notes on Number Theory and Discrete Mathematics. Vol. 21(3) 1-21 (2015).

[7] Biswas, Ranjit.: Region Mathematics - A New Direction In Mathematics: Part-2. Pure and Applied Mathematics Journal (communicated).

[8] Biswas, Ranjit.: Is 'Fuzzy Theory' An Appropriate Tool For Large Size Problems ?. in the book-series of SpringerBriefs in Computational Intelligence. Springer. Heidelberg. (2016)

[9] Biswas, Ranjit.: Is 'Fuzzy Theory' An Appropriate Tool For Large Size Decision Problems ?, Chapter- 8 in Imprecision and Uncertainty in Information Representation and Processing, in the series of STUDFUZZ. Springer. Heidelberg. (2016)

[10] Beachy, J. A., and Blair, W. D.: Abstract Algebra. 2nd Ed., Waveland Press, Prospect Heights, Ill. (1996)

[11] Bourbaki, Nicolas.:Elements of Mathematics: Algebra I.New York: Springer-Verlag. (1998)

[12] Copson, E. T.: Metric Spaces. Cambridge University Press (1968)

[13] Dixon, G. M.: Division Algebras: Octonions Quaternions Complex Numbers and the Algebraic Design of Physics. Kluwer Academic Publishers, Dordrecht. (2010)

[14] Ellis, G.: Rings and Fields. Oxford University Press. (1993)

[15] Herstein, I. N.: Topics in Algebra. Wiley Eastern Limited. New Delhi. (2001)

[16] Hungerford, T., Algebra, Graduate Texts in Mathematics, Vol. 73, Springer-Verlag, New York, 1974.

[17] Jacobson, N.: Basic Algebra I. 2nd Ed., W. H. Freeman \& Company Publishers, San Francisco. (1985)

[18] Jacobson, N.: Basic Algebra II. 2nd Ed., W. H. Freeman \& Company Publishers, San Francisco. (1989) 
[19] Jacobson, N.: The Theory of Rings. American Mathematical Society Mathematical Surveys. Vol. I. American Mathematical Society. New York. (1943)

[20] Lam, T. Y.: Exercises in Classical Ring Theory, Problem Books in Mathematics, Springer-Verlag, New York. (1995)

[21] Lang, Serge.: Undergraduate Algebra. (3rd ed.). Berlin, New York: Springer-Verlag. (2005)

[22] Matsumura, Hideyuki.: Commutative Ring Theory. Cambridge University Press, Cambridge. (1986)

[23] Pierce, Richard S.: Associative algebras. Graduate Texts in Mathematics. Studies in the History of Modern Science. Springer-Verlag. Berlin. (1982)
[24] Reyes, Mitchell.: The Rhetoric in Mathematics: Newton, Leibniz, the Calculus, and the Rhetorical Force of the Infinitesimal. Quarterly Journal of Speech. Vol. 90. 159-184 (2004).

[25] Rudin, W alter.: Real and Complex Analysis. McGraw Hills Education, India. (2006)

[26] Saltman, D. D.: Lectures on Division Algebras. Providence, RI: Amer. Math. Society. (1999)

[27] Simmons, G. F.: Introduction to Topology and Modern Analysis. McGraw Hill, New York. (1963)

[28] Van der Waerden and BartelLeendert.: Algebra. SpringerVerlag, New York. (1991) 